\title{
Evaluación de la hipótesis de eficiencia débil y análisis de causalidad en las centrales de abastos de Colombia
}

\author{
Testing the Weak Form of Efficient Market Hypothesis and Causality \\ Analysis in Colombian Food Supply Centers
}

Avaliação da hipótese da eficiência fraca do mercado e análise de
causalidade em centros de abastecimento de comida da Colômbia

\author{
Leonardo Hernán Talero-Sarmiento* \\ Henry Lamos-Díaz** \\ Edwin Alberto Garavito-Hernández***
}

DOI: https://doi.org/10.19053/01203053.v38.n67.2019.8040

Fecha de recepción: 20 de marzo de 2017

Fecha de aprobación: 25 de mayo de 2017

Cómo citar este artículo/ to reference this article / comment citer cet article / para citar este artigo:

Talero-Sarmiento, L., Lamos-Díaz, H. \& Garavito-Hernández, E. (2019). Evaluación de la hipótesis de eficiencia débil y análisis de causalidad en las centrales de abastos de Colombia. Apuntes del Cenes, 38(67). https://doi.org/10.19053/01203053.v38.n67.2019.8040

* Magíster en Ingeniería Industrial de la Universidad Industrial de Santander. Investigador de la Escuela de Estudios Industriales y Empresariales, Universidad Industrial de Santander. Bucaramanga, Colombia. leonardo.talero@correo.uis.edu.co (1) https://orcid.org/0000-0002-4129-9163

** Doctor en Matemática Aplicada de la Universidad Estatal de Moscú. Profesor titular de la Escuela de Estudios Industriales y Empresariales, Universidad Industrial de Santander. Bucaramanga, Colombia. hlamos@uis.edu.co (1) https://orcid. org/0000-0003-1778-9768

***Magíster en Ingeniería Industrial de la Universidad Mayaguez de Puerto Rico. Profesor asociado de la Escuela de Estudios Industriales y Empresariales, Universidad Industrial de Santander. Bucaramanga, Colombia. garavito@uis.edu.co (1D https:// orcid.org/0000-0002-0145-232X 
Evaluación de la hipótesis de eficiencia débil y análisis de causalidad en las centrales de abastos de Colombia Leonardo Hernán Talero-Sarmiento - Henry Lamos-Díaz - Edwin Alberto Garavito-Hernández

\section{Resumen}

En Colombia existen centros tradicionales de acopio donde se comercializan y distribuyen productos agrícolas. El precio de venta de estos productos es publicado por instituciones del Gobierno en informes semanales. Con el objetivo de determinar si las series de precios siguen una caminata aleatoria y si existen relaciones entre los centros, se aplican seis pruebas que evalúan la hipótesis de eficiencia débil y la prueba de causalidad de Granger. Para ello se analiza el precio histórico semanal de 28 productos agrícolas comercializados en seis mercados, durante la primera semana de 2013 a la última de 2017. Los principales resultados indican que los mercados tienden a la eficiencia, aunque no tienen el mismo nivel, ya que esta varía según el producto que se comercialice. Además, los centros en Manizales, Barranquilla y Villavicencio influyen sobre los precios de los mercados de Bogotá, Bucaramanga y Medellín.

Palabras clave: prueba de hipotesis, causalidad de Granger, mercados agrícolas, microeconomía, precios agrícolas.

Clasificación JEL: C12, D4, D40, G14, Q13. 


\section{Abstract}

In Colombia, there are traditional food supply centers to trade agricultural products. Government institutions publish sale prices of these products in weekly reports. In order to determine the random characteristics of these price series and if there are relationships between the centers, we use a battery of six different tests to examine the weak form of efficient market hypothesis and the Granger causality test. For this, this work collected the historical weekly price of 28 agricultural products, taking into account six markets, during the first week of 2013 to the last week of 2017. The main results indicate that markets tend towards efficiency, however, the efficiency depends on the product traded. In addition, the centers in Manizales, Barranquilla and Villavicencio influence the prices of the Bogotá, Bucaramanga and Medellín markets.

Keywords: Hypotesis testing, Granger causality, agricultural markets, microeconomics, agricultural prices. 
Evaluación de la hipótesis de eficiencia débil y análisis de causalidad en las centrales de abastos de Colombia Leonardo Hernán Talero-Sarmiento - Henry Lamos-Díaz - Edwin Alberto Garavito-Hernández

\section{Resumo}

Na Colômbia, existem centros de coleta tradicionais, onde os produtos agrícolas são comercializados e distribuídos, o preço de venda desses produtos é publicado por instituições governamentais em relatórios semanais. Para determinar se as séries de preços seguem um passeio aleatório e se existem relações entre os centros, são aplicados seis testes que avaliam a hipótese de eficiência fraca e o teste de causalidade de Granger. Para tanto, é analizado o preço semanal histórico de vinte e oito produtos agrícolas comercializados em seis mercados, durante a primeira semana de 2013 até a última semana de 2017. Os principais resultados indicam que os mercados tendem para a eficiência, embora não tenham o mesmo nível porquanto a eficiência varia de acordo com o produto que é comercializado. Além disso, os centros de Manizales, Barranquilla e Villavicencio influenciam os preços dos mercados de Bogotá, Bucaramanga e Medellín.

Palavras-chave: Teste de hipóteses, causalidade de Granger, mercados agrícolas, microeconomia, preços agrícolas. 


\section{INTRODUCCIÓN}

El sector agropecuario es de gran importancia para el crecimiento económico de los países, por ser fuente de alimentos y materias primas, y generar empleo y divisas (Cárdenas \& Vallejo, 2016). En Colombia, los ingresos derivados de las actividades agropecuarias representan más del 6 $\%$ del producto interno bruto (PIB) (Departamento Administrativo Nacional de Estadística [DANE], 2017); no obstante, su crecimiento es inferior al nacional e históricamente ha caído desde un $9 \%$, lo cual refleja una reducción en el precio relativo de la producción agropecuaria medida a través de los deflactores del PIB (Departamento Nacional de Planeación [DNP], 2015). Además, si bien el sector mantiene una balanza comercial positiva, esta no es estática, ya que las importaciones han venido aumentando a un ritmo más acelerado que las exportaciones (Gómez, 2011), lo cual afecta el crecimiento del valor agregado de los subsectores que lo componen, es decir, como consecuencia de la relación entre importaciones-exportaciones en el sector agropecuario, productos como el sorgo, la cebada y el trigo presentan una caída importante, casi desapareciendo de la composición del PIB sectorial, mientras que productos de ciclo corto y la mayoría de cultivos permanentes, sin considerar el café, han aumentado su nivel de producción (DNP, 2015), por tanto, es importante estudiar los precios de la producción agropecuaria no transable ya que esta ha ido en aumento. 
Por otra parte, en Colombia existen centros tradicionales de acopio, denominados centrales de abastos (CA), donde se comercializan y distribuyen productos agrícolas. Estos centros son de gran importancia, ya que se enfocan en el mercado a nivel nacional, y el comercio de productos agrícolas en el interior de un país y en zonas periurbanas no solo aumenta la cobertura alimentaria (Toth, Rendall \& Reitsma, 2016), sino ayuda a disminuir la volatilidad de los precios (Minot, 2014), puesto que estos dependen menos de la importación de alimentos y, por tanto, existe una menor transferencia entre el mercado global y el local (Bekkers, Brockmeier, Francois \& Yang, 2017), lo cual aumenta la soberanía alimentaria (Food and Agriculture Organization of the United Nations [FAO], 2011).

Teniendo en cuenta el aumento de productos agrícolas no transables y que estos pueden ser comercializados en centrales de abastos, es posible analizar la variación de los precios de venta en dichos mercados a partir de diversas características, como tendencia, estacionariedad y aleatoriedad, mediante la evaluación de la hipótesis de eficiencia débil y análisis de causalidad (ambas pruebas pueden ser aplicadas con el histórico de los precios de venta), lo cual permite determinar si existe alguna relación en la manera en la cual se generan los precios entre las centrales de abastos.
El desarrollo de este tipo de estudios es de interés para diversos inversores y productores, ya que entender la forma en que varían los precios de productos agrícolas ayuda a fortalecer políticas públicas de inversión y genera confianza en los mercados donde estos son comercializados (Karali \& Power, 2013).

En concordancia con la importancia previamente descrita, en la literatura científica se encuentran diversos estudios de las series de precios de productos agropecuarios, de los cuales se identifican dos objetivos. Por un lado están aquellos que aplican modelos de predicción para estimar el retorno de la inversión o los precios de venta $y$, por otra parte, aquellos que buscan evaluar hipótesis sobre la naturaleza de las series o los mercados en donde son comercializados los productos; estos últimos aportan al primer enfoque en cuestión, ya que entender la dinámica de los precios ayuda a generar pronósticos acertados (Malkiel, 2003). No obstante, es importante resaltar que estos trabajos se enfocan solamente en los mercados bursátiles y que, durante la presente investigación, no fueron encontradas referencias de trabajos equivalentes a centrales de acopio y venta; por tanto, desde un aspecto teórico, se logra la aplicación de estas propuestas econométricas en un mercado diferente al bursátil (y en extensión a productos 
no transables), lo cual genera bases para futuros trabajos relacionados con la hipótesis de eficiencia semifuerte y fuerte, así como las aplicaciones e implicaciones que estas conllevan.

Considerando lo anterior, el presente artículo se enfoca en el análisis de relaciones entre las centrales de abastos mediante un estudio de causalidad de precios, así como en evaluar la hipótesis de eficiencia de los mercados en su variante débil (ya que mediante las pruebas aplicadas es posible identificar modelos autorregresivos estacionarios para usar en el estudio de causalidad). Para ello, el documento se organiza de la siguiente forma: en la primera parte del trabajo (Antecedentes) se exponen algunos estudios relacionados con la hipótesis de eficiencia de los mercados y el análisis series de precios de venta de productos agrícolas; posteriormente, en la sección de Materiales y Métodos, se explica de forma breve cómo se seleccionaron los productos y los respectivos mercados de abastos, y se exponen las siete pruebas aplicadas. En Resultados se analizan las hipótesis aceptadas y rechazadas; en la sección de Discusión se mencionan ciertas limitaciones encontradas durante el desarrollo de la investigación, y en la parte final del trabajo se presentan las conclusiones, las referencias y los anexos.

\section{ANTECEDENTES}

\section{Estudios relacionados con la hipótesis de eficiencia débil}

Dentro de los estudios enfocados en el análisis de la variabilidad de los precios se encuentra el trabajo desarrollado por Levy (1967), quien es posiblemente el primer autor en diferenciar la forma fuerte y débil de la hipótesis de eficiencia de los mercados (HEM) propuesta por Fama (1965). Levy indica que el nivel de eficiencia depende de las características de aleatoriedad, puesto que, ante la presencia de comportamientos como las caminatas aleatorias no es factible pronosticar precios, por tanto, no se pueden obtener rendimientos extraordinarios mediante modelos como los autorregresivos. El presente trabajo se enfoca en la variante débil de la hipótesis de eficiencia de los mercados, ya que el concepto de eficiencia ha sido cuestionado principalmente en su forma fuerte, como consecuencia del costo de la información y las asimetrías que este puede generar (Grossman \& Stiglitz, 1980), cuestionamiento que no suele aplicarse en las otras formas, debido a que la información requerida suele ser pública y gratuita (Fama, 1991), como la utilizada en el presente trabajo.

Ahora bien, es importante resaltar que evaluando la hipótesis de eficiencia de los mercados se pueden obtener 
resultados contradictorios o no concluyentes, ya que, dependiendo del horizonte del análisis, el mercado estudiado y las pruebas aplicadas es posible aceptar, rechazar o no concluir (cuando una prueba contradice a otra). Como antecedentes relacionados con la hipótesis de eficiencia de los mercados se encuentra que de los trabajos empíricos publicados entre 1997 a 2012 en Science Direct, el $60 \%$ niega esta hipótesis, el $35 \%$ no la rechaza y un $5 \%$ de los estudios indica una mejora en la eficiencia (Duarte-Duarte \& Mascareñas, 2014b), por tanto, es recomendable que al tratar con más de una prueba en el mismo horizonte de estudio se considere la potencia de las mismas.

Debido a las distintas discusiones que pueden darse en torno a la evaluación de la hipótesis de eficiencia de los mercados, la aplicación de pruebas sigue siendo un tema vigente. De hecho, en Latinoamérica se encuentran trabajos realizados por Duarte-Duarte y Mascareñas, quienes determinan mediante un estudio comprendido entre 2002 y 2012 que las principales economías en la región han presentado un aumento en su eficiencia (2014a); no obstante, esos resultados contrastan con los trabajos posteriores de Talero, Duarte y Garcés (2017), quienes, a partir de métodos de simulación, evalúan dichos mercados entre 2004 y 2016, y encuentran una disminución en la eficiencia con comportamientos leptocúrticos en el retorno de sus activos, lo que implica que las series no siempre siguen una caminata aleatoria y a veces presentan persistencia; lo cual, según un análisis para Colombia, puede ser consecuencia de la composición de los mercados, ya que es posible que coexistan diferentes tipos de agentes y su porcentaje de participación explique la eficiencia o persistencia de las series (Duarte, Talero \& Sierra, 2017). Entre otros trabajos relacionados con la hipótesis de eficiencia de los mercados está el desarrollado por Ruiz-Porras y Ruiz-Robles (2015), quienes señalan que el mercado mexicano presenta una disminución en su eficiencia débil posterior al año 2007. De manera general, la aceptación de la hipótesis de eficiencia de los mercados puede indicar que existen condiciones justas para comercializar en los mercados bajo estudio, lo cual puede ser explicado por 1) reformas en las economías que buscan liberalización o menos restricciones al mercado, 2) mayor velocidad en el flujo de la información posibilitado por internet, 3) introducción de nuevos productos financieros y 4) periodos poscrisis económicas y bursátiles, que llevan a corregir asimetrías del mercado (Duarte-Duarte \& Mascareñas, 2014b).

\section{Estudios sobre la variabilidad de precios de productos agrícolas}

En la literatura científica es posible encontrar diversos trabajos enfocados 
en evaluar la eficiencia, memoria o variabilidad de diversas series de precios de productos agropecuarios, con el propósito de generar modelos de predicción de precios más acertados. Respecto al estudio sobre productos colombianos, se puede encontrar un trabajo realizado por Velásquez y Aldana (2007), quienes abordan los dos enfoques (estudio de la serie y predicción). Los autores analizan el precio promedio mensual del café colombiano en la Bolsa de Nueva York usando varios modelos alternativos, tanto autorregresivos como máquinas de aprendizaje; proponen un modelo compuesto por una componente lineal autorregresiva más una red neuronal artificial tipo perceptrón multicapa con dos neuronas en la capa oculta, y a partir de los hallazgos concluyen que el precio actual depende de los precios ocurridos en los últimos cuatro meses (indican que el mercado no presenta un comportamiento eficiente).

Años más tarde, Coronado, Ramírez y Celso (2014) estudian la dependencia no lineal en los retornos del precio del café colombiano arábico (Colombian Arabica Coffee) utilizando el test estadístico de Hinich portmanteau (H), en el periodo comprendido entre junio 29 de 1990 y julio 1 de 2010. Los autores encuentran que en el $5.78 \%$ de los casos, los modelos de volatilidad como GARCH no logran capturar los comportamientos de no linealidad, lo que indica que no puede aceptarse la hipótesis de eficiencia del mercado en su forma débil. Posteriormente, Coronado et al. (2014) evalúan la hipótesis de mercado adaptativo propuesta por Andrew Lo (2005) para ocho diferentes productos agrícolas catalogados como commodities, mediante pruebas no lineales y análisis de bicorrelación.

Respecto al análisis de otros productos agropecuarios, Tansuchat, Chang y McAleer (2009) desarrollan un trabajo en el cual evalúan el nivel de memoria a largo plazo de diversos productos agropecuarios que cotizan en bolsa: maíz, avena, soja, harina de soja, aceite de soja, trigo, ganado vivo, alimentos para ganado, carne de cerdo, cacao, café, algodón y jugo de naranja, utilizando modelos GARCH, FIGARCH, FIEGARCH, FIAPARCH, EGARCH y APARCH, y encuentran que a largo plazo existe dependencia en los precios estudiados. En ese mismo año, Benavides (2004) presenta un trabajo que se enfoca en el contraste entre modelos (teniendo en cuenta el error cuadrado medio del pronóstico como criterio de evaluación), como el modelo GARCH, ARCH (modelo BEKK) y modelos de volatilidad, los cuales son aplicados a las series de precios del maíz y avena, y encuentran que un modelo compuesto es el que presenta mejor ajuste. Por otra parte, Tejeda y Goodwin (2009) aplican un modelo de series de tiempo multivariante, con el 
cual evalúan correlaciones asimétricas entre los precios del grano (maíz, soya) y del ganado, y analizan la relación entre el volumen de producción de maíz y el precio del etanol; hallan una relación negativa entre los precios del maíz y de los becerros con el volumen de producción de etanol, lo cual implica una estacionalidad en las series de precios.

Años más tarde, Lachman y Jack (2017) estudian la eficiencia en los mercados de futuros de la soya entre los mercados de Buenos Aires (MatBa) y Chicago (CME-CBOT) para los años 1994 a 2015. Aceptando la hipótesis de eficiencia del mercado en MatBa, la cual se relaciona con la eficiencia en el mercado de CMECBOT, es decir, a partir de la prueba propuesta por Johansen mediante series de precios mensuales y diarios existe cointegración entre los mercados. De manera paralela, Bouri, Chang y Gupta (2017) evalúan la hipótesis de eficiencia del mercado en su forma débil para el mercado del vino, utilizando una versión modificada de la prueba de raíz unitaria. Observan cambios estructurales en la serie, así como no linealidad, además concluyen que contrario a los resultados de la prueba tradicional, con la prueba de raíz unitaria propuesta pueden demostrar que el mercado es ineficiente.
Teniendo en cuenta los trabajos citados previamente, es posible determinar una estructura metodológica. Como primer paso es necesario evaluar la hipótesis de eficiencia de los mercados en su forma débil, puesto que si los mercados no son eficientes, estos pueden presentar asimetrías de información, lo cual puede ser reflejado en la volatilidad de las series; caso contrario, al no rechazar esta hipótesis se pueden aplicar transformaciones a las series para obtener características estacionarias mediante modelos lineales y, con ello, realizar comparaciones entre los mercados (CA) mediante pruebas de causalidad o cointegración, esto permite en el futuro el desarrollo de herramientas para la toma de decisiones.

\section{MATERIALES Y MÉTODOS}

\section{Datos}

Los datos son obtenidos a partir de los reportes semanales generados por el Sistema de Información de Precios y Abastecimientos del Sector Agropecuario (SIPSA), el cual es una división del Departamento Administrativo Nacional de Estadística (DANE) de Colombia. De cada reporte semanal generado durante el periodo comprendido entre enero de 2013 (cuando unifican reportes semiestructurados en formato Excel) y diciembre de 2017 (fecha en la cual fue realizado el 
análisis), se extrae de manera estructurada el precio promedio de venta por unidad de kilo $[\mathrm{COP} / \mathrm{Kg}]$ de 216 productos divididos en cuatro categorías: hortalizas: 74 , frutas frescas: 76 , raíces y tubérculos: 39 , y granos y cereales: 28. Datos recolectados para 12 centrales de abastos: Barranquilla, Bogotá, Bucaramanga, Cali, Cartagena, Ipiales, Manizales, Medellín, Montería, Neiva, Sincelejo y Villavicencio.

Una vez estructurados en tablas los precios históricos de los productos agrícolas, se revisa la ausencia de datos (i.e. datos perdidos), y se seleccionan aquellos productos que presentan a lo sumo un $10 \%$ de datos perdidos y que tengan presencia en todas las centrales de abastos; sin embargo, debido a la ausencia de registros en varias centrales, se simplifica la cantidad de productos y su punto de venta, generando así un histórico de 621 entradas en cada mercado (central de abastos) para un total de 28 productos comercializados en seis centrales de abastos (Barranquilla, Bogotá Bucaramanga, Manizales, Medellín y Villavicencio).
Los productos estudiados se enumeran en la Tabla 1, donde se agrupan los 28 productos analizados según la categoría identificada en los reportes del SIPSA; así mismo, se indica la cantidad porcentual de datos perdidos para cada una de las centrales de abastos estudiadas, donde nueve productos no presentan datos perdidos, ocho productos indican datos perdidos en una única central de abastos, cinco productos en dos centrales, tres productos muestran datos perdidos en tres centrales y un único producto tiene datos perdidos en las seis centrales. Además, se evidencia que pocos productos tienen una cantidad relativamente alta de datos perdidos (dos productos no registran 22 precios, y un producto, 25).

Posteriormente, y teniendo en cuenta la baja cantidad de datos perdidos, estos son imputados a partir de la interpolación simple entre los datos presentes, considerando que en la mayoría de los casos la ausencia de datos era escasa y también en la mayoría de los casos faltaba un único registro de manera consecutiva. 
Evaluación de la hipótesis de eficiencia débil y análisis de causalidad en las centrales de abastos de Colombia Leonardo Hernán Talero-Sarmiento - Henry Lamos-Díaz - Edwin Alberto Garavito-Hernández

Tabla 1. Proporción de datos perdidos según categoría

\begin{tabular}{|c|c|c|c|c|c|c|c|c|}
\hline \multirow[b]{2}{*}{ Cód. } & \multirow[b]{2}{*}{ Categoría } & \multirow[b]{2}{*}{ Nombre } & \multicolumn{6}{|c|}{ Datos perdidos } \\
\hline & & & $\mathrm{Ba} / \mathrm{lla}$ & Bo/tá & B/ga & Ma/les & Me/llín & $\mathrm{Vi} /$ cio \\
\hline $\mathrm{p} 1$ & $\mathrm{R} \& \mathrm{~T}$ & Apio & $0,00 \%$ & $0,00 \%$ & $0,00 \%$ & $0,00 \%$ & $0,00 \%$ & $0,00 \%$ \\
\hline p2 & $\mathrm{H}$ & Brócoli & $0,00 \%$ & $0,00 \%$ & $0,00 \%$ & $0,00 \%$ & $0,00 \%$ & $0,00 \%$ \\
\hline p3 & $\mathrm{G} \& \mathrm{C}$ & Chócolo mazorca & $0,00 \%$ & $0,00 \%$ & $0,00 \%$ & $3,07 \%$ & $0,00 \%$ & $0,00 \%$ \\
\hline $\mathrm{p} 4$ & $\mathrm{H}$ & Cilantro & $0,00 \%$ & $0,00 \%$ & $1,15 \%$ & $0,77 \%$ & $0,00 \%$ & $0,00 \%$ \\
\hline p5 & $\mathrm{H}$ & Coliflor & $0,00 \%$ & $9,58 \%$ & $0,00 \%$ & $0,38 \%$ & $0,00 \%$ & $0,00 \%$ \\
\hline p6 & $\mathrm{H}$ & Habichuela & $0,00 \%$ & $0,00 \%$ & $0,00 \%$ & $0,00 \%$ & $0,00 \%$ & $0,00 \%$ \\
\hline p7 & $\mathrm{H}$ & Lechuga batavia & $0,00 \%$ & $0,38 \%$ & $0,00 \%$ & $0,00 \%$ & $0,00 \%$ & $0,00 \%$ \\
\hline $\mathrm{p} 8$ & $\mathrm{H}$ & Pepino cohombro & $0,00 \%$ & $0,00 \%$ & $0,00 \%$ & $0,00 \%$ & $0,00 \%$ & $0,00 \%$ \\
\hline p9 & $\mathrm{H}$ & Pimentón & $0,00 \%$ & $0,00 \%$ & $0,00 \%$ & $0,00 \%$ & $0,00 \%$ & $0,00 \%$ \\
\hline p10 & $\mathrm{FF}$ & Guayaba pera & $0,38 \%$ & $0,00 \%$ & $0,00 \%$ & $0,00 \%$ & $0,00 \%$ & $0,00 \%$ \\
\hline p11 & FF & Lulo & $0,00 \%$ & $0,00 \%$ & $0,00 \%$ & $0,38 \%$ & $0,00 \%$ & $0,00 \%$ \\
\hline $\mathrm{p} 12$ & FF & Manzana roja importada & $0,38 \%$ & $1,53 \%$ & $0,00 \%$ & $0,77 \%$ & $0,00 \%$ & $0,77 \%$ \\
\hline p13 & FF & $\begin{array}{l}\text { Manzana verde } \\
\text { importada }\end{array}$ & $1,15 \%$ & $2,68 \%$ & $0,00 \%$ & $0,00 \%$ & $0,00 \%$ & $0,00 \%$ \\
\hline p14 & FF & Mora de Castilla & $0,00 \%$ & $0,00 \%$ & $0,00 \%$ & $0,00 \%$ & $0,00 \%$ & $0,00 \%$ \\
\hline p15 & $\mathrm{FF}$ & Papaya Maradol & $4,60 \%$ & $0,00 \%$ & $0,00 \%$ & $0,00 \%$ & $0,00 \%$ & $0,00 \%$ \\
\hline p16 & $\mathrm{FF}$ & Tomate de árbol & $0,00 \%$ & $0,00 \%$ & $0,00 \%$ & $0,00 \%$ & $0,00 \%$ & $0,00 \%$ \\
\hline p17 & $\mathrm{G} \& \mathrm{C}$ & Arroz de primera & $0,00 \%$ & $0,00 \%$ & $0,38 \%$ & $0,00 \%$ & $0,38 \%$ & $0,38 \%$ \\
\hline p18 & G\&C & Lenteja importada & $0,00 \%$ & $0,00 \%$ & $0,00 \%$ & $0,38 \%$ & $0,38 \%$ & $1,53 \%$ \\
\hline p19 & $\mathrm{H}$ & Espinaca & $0,00 \%$ & $0,00 \%$ & $0,00 \%$ & $0,00 \%$ & $0,00 \%$ & $0,00 \%$ \\
\hline p20 & $\mathrm{H}$ & Remolacha & $0,00 \%$ & $0,00 \%$ & $0,00 \%$ & $0,38 \%$ & $0,00 \%$ & $0,00 \%$ \\
\hline $\mathrm{p} 21$ & FF & Granadilla & $8,43 \%$ & $0,00 \%$ & $0,38 \%$ & $2,68 \%$ & $0,00 \%$ & $0,38 \%$ \\
\hline p22 & FF & Melón Cantalup & $0,38 \%$ & $0,00 \%$ & $0,00 \%$ & $0,00 \%$ & $0,77 \%$ & $0,00 \%$ \\
\hline p23 & $\mathrm{FF}$ & Uva red globe nacional & $1,15 \%$ & $0,00 \%$ & $0,77 \%$ & $0,00 \%$ & $0,00 \%$ & $8,43 \%$ \\
\hline p24 & $\mathrm{H}$ & Papa criolla limpia & $2,30 \%$ & $0,00 \%$ & $0,00 \%$ & $0,00 \%$ & $0,00 \%$ & $0,00 \%$ \\
\hline p25 & $\mathrm{G} \& \mathrm{C}$ & $\begin{array}{l}\text { Arveja verde seca } \\
\text { importada }\end{array}$ & $1,15 \%$ & $0,38 \%$ & $0,38 \%$ & $1,15 \%$ & $0,77 \%$ & $1,53 \%$ \\
\hline p26 & $\mathrm{H}$ & Arveja verde en vaina & $0,00 \%$ & $0,00 \%$ & $0,38 \%$ & $3,07 \%$ & $0,00 \%$ & $0,00 \%$ \\
\hline p27 & $\mathrm{H}$ & $\begin{array}{l}\text { Cebolla cabezona } \\
\text { blanca }\end{array}$ & $0,00 \%$ & $0,00 \%$ & $0,00 \%$ & $0,00 \%$ & $0,00 \%$ & $0,00 \%$ \\
\hline p28 & FF & Naranja Valencia & $0,38 \%$ & $0,00 \%$ & $0,00 \%$ & $0,00 \%$ & $0,00 \%$ & $0,00 \%$ \\
\hline
\end{tabular}

Ba/lla: Barranquilla, Bo/tá: Bogotá, B/ga: Bucaramanga, Ma/les: Manizales, Me/lí́n: Medellín, Vi/cio: Villavicencio, R\&T: raíces y tubérculos, H: hortalizas, G\&C: granos y cereales, FF: frutas frescas. 


\section{Métodos para la estimación de aleatoriedad y prueba de causalidad}

El desarrollo metodológico se enfoca en el formato de estudio empírico utilizando fuentes secundarias de datos, donde estos son analizados mediante la aplicación de seis pruebas que permiten discernir si las series de precios son de carácter estacionario o no y una prueba para analizar causalidad. Para ello, los datos son transformados de series de precios a series de rentabilidad, lo cual permite medir el retorno en un momento específico, según la siguiente ecuación:

$$
R_{t}-\ln \left(P_{t} / P_{t-1}\right)
$$

Donde $R_{t}$ es el retorno esperado en el instante $t, \ln$ es el logaritmo natural, $P_{t}$ es el precio promedio de venta del producto agrícola durante la semana y $P_{t-1}$ el precio de venta promedio de dicho producto en la semana anterior.

\section{Prueba de raíz unitaria}

Las primeras tres pruebas aplicadas durante el presente trabajo pertenecen a la familia de las raíces unitarias, las cuales, según citan Nisar y Hanif (2012, p. 418), "pueden ser usadas para evaluar la eficiencia de los mercados, puesto que dicho comportamiento demanda aleatoriedad (i.e. serie no estacionaria) en los precios (Hassan, Abdullah, \& Shah,
2007)". Estas son evaluadas utilizando el paquete "urca" desarrollado en el software estadístico R por Pfaff (2008).

\section{Raíz unitaria con deriva y con tendencia}

La primera prueba se enfoca en determinar si el modelo general de regresión se comporta como el descrito en la ecuación [2]:

$$
Y_{t}=\beta_{1}+\beta_{2} t+\beta_{3} Y_{t-1}+u_{t}
$$

Donde $u_{t}$ es un término de error de ruido blanco (se distribuye normalmente con media $=0$ y varianza $=1), t$ es el tiempo medido cronológicamente, $Y_{t}$ la realización de la serie, $\beta_{1}$ indica el valor de deriva, $\beta_{2}$ la tendencia temporal y $\beta_{3}$ la raíz del proceso. Con la característica de presentar un $-1 \leq \beta_{3} \geq 1$ Donde si $\beta_{3}$ $=1$ se conoce como raíz unitaria que indica un modelo de caminata aleatoria $\mathrm{y}$, por tanto, se entiende que es un proceso estocástico no estacionario. Como hipótesis nula se evalúa que el modelo de regresión presenta un problema de raíz unitaria $H_{0}{ }^{l}: \beta_{1} \neq 0, \beta_{2} \neq 0, \beta_{3}=1$; $H_{1}^{l}$ :Caso contrario.

Raíz unitaria con deriva y sin tendencia

Del modelo general de regresión se puede presentar una forma especial en la cual $\beta_{1} \neq 0, \beta_{2}=0, \beta_{3}=1$ :

$$
Y_{t}=\beta_{1}+\beta_{3} Y_{t-1}+u_{t}
$$


Dicho modelo se conoce como caminata aleatoria con deriva (CAD), que se caracteriza por ser no estacionaria con tendencia determinista. Como hipótesis nula se evalúa que el modelo de regresión presenta un problema de raíz unitaria con tendencia, es decir: $H_{0}^{2}: \beta_{1} \neq 0, \beta_{2}=0, \beta_{3}=1 ; H_{1}^{2}:$ Caso contrario.

\section{Raiz unitaria sin deriva y sin tendencia}

Del modelo general de regresión se puede presentar una forma especial en la cual $\beta_{1}=0, \beta_{2}=0, \beta_{3}=1$

$$
Y_{t}=Y_{t-1}+u_{t}
$$

El modelo es conocido como caminata aleatoria pura (CAP), el cual genera un proceso no estacionario. Como hipótesis nula se evalúa que el modelo de regresión presenta un problema de raíz unitaria con tendencia, es de$\operatorname{cir}: H_{0}^{3}: \beta_{1}=0, \beta_{2}=0, \beta_{3}=1 ; H_{1}^{3}:$ Caso contrario.

\section{Prueba de rachas (prueba de Geary)}

El cuarto test aplicado es una prueba no paramétrica que busca determinar si las realizaciones en una serie siguen un comportamiento aleatorio (Geary, 1935; Gujarati \& Porter, 2010), para ello se estima un valor crítico $Z$ teniendo en cuenta la siguiente ecuación:

$$
Z=R-\left(X / \sigma^{2}\right)
$$

Donde $Z$ es una variable que se distribuye de manera normal, $R$ es el número total de rachas, $X=\left(2 n^{l} n^{2}\right) /(n)$ +1 (media de la serie), $n^{l}$ representa el número total de rachas positivas, $n^{2}$ el número total de rachas negativas, $n=\left(n^{1}+n^{2}\right), \quad \sigma 2=\left(2 n^{l} n^{2}\left(2 n^{l} n^{2}-n\right)\right)$ $\left((n)^{2}(n-1)\right)$ (varianza de la serie).

A partir del cálculo de la ecuación [5] se obtiene un estadístico, si su valor se encuentra entre el rango $(-1.96,1.96)$ - a un $95 \%$ de confianza- indicará que la serie presenta un comportamiento aleatorio según sus realizaciones. Como hipótesis nula se evalúa que el valor obtenido de $Z$ representa una serie de realizaciones aleatorias, es decir: $\mathrm{H}_{0}^{4}:-1.96<\mathrm{Z}<1.96 ; \mathrm{H}_{1}^{4}: \mathrm{Ca}$ so contrario. Esta prueba es evaluada utilizando el paquete "randtest" desarrollado en el software estadístico R por Caeiro y Mateus (2014).

\section{Análisis de autocorrelaciones (prueba d, de Durbin-Watson)}

La quinta prueba es el análisis de autocorrelaciones de Durbin-Watson (Savin \& White, 1977), que se utiliza para detectar la presencia de autocorrelación en los residuos (errores de predicción) de un análisis de la regresión. El estadístico es calculado a continuación: 


$$
\begin{gathered}
d=\left(\sum_{(t=2)}^{T}\left(e_{t}-e_{(t-1)}\right)^{2}\right) / \\
\left(\sum_{(t=1)}^{T}\left(e_{t}\right)^{2}\right)
\end{gathered}
$$

Donde

$$
d \approx\left(2-\beta_{3}\right)
$$

Indicando que $\beta_{3}$ representa el grado de correlación de la muestra de los residuos. $d=2$ indica que no hay autocorrelación (Durbin \& Watson, 1951). Como hipótesis nula se evalúa que no existe autocorrelación en la serie, es decir: $H_{0}^{5}: P(d=2) ; H_{1}^{5}:$ Caso contrario. En el presente trabajo es utilizado el paquete estadístico "Imtest" desarrollado en $\mathrm{R}$ por Zeileis y Hothorn (2002).

\section{Estimación del coeficiente de Hurst}

La sexta prueba es la estimación del coeficiente de Hurst, medida que indica el grado de independencia de las series de tiempo. Cuando el valor del coeficiente se encuentre en $H=0.5$, se considera que la serie tiene un comportamiento de ruido blanco. Por otra parte, si $(0.5 \leq H<1]$ se dice que la serie presenta persistencia o autocorrelaciones, de manera análoga, si $[0 \leq H \leq 0.5)$ la serie presenta un comportamiento antipersistente o de correlación negativa.

Para el presente trabajo es aplicada la variante propuesta por Andrew Lo
(1991), la cual tiene en cuenta la memoria interna de la serie, donde $\sigma_{\mathrm{x}}^{2}$ y $\hat{\mathrm{Y}}_{\mathrm{j}}$ son los estimadores de varianza $\mathrm{y}$ covarianza de la serie, $a$ el tamaño de la subserie, $R(N)$ el rango de datos de la subserie.

$$
\begin{gathered}
F^{\prime}(N)=R(N) / \\
\left(\left(\sigma_{x}^{2}+2 \sum_{(j=1)}^{a}(1-j /(a+1))^{*} \hat{y}_{j}\right)^{(1 / 2))}\right.
\end{gathered}
$$

Como hipótesis nula se evalúa si la serie presenta un comportamiento aleatorio, es decir: $H_{0}^{6}: H=0.5 ; H_{1}^{6}:$ Caso contrario. Ahora bien, teniendo en cuenta que el coeficiente de Hurst es una estimación y es sensible a la longitud de la serie y la cantidad de subseries (Talero et al., 2017), es necesario determinar el número de subseries con su respectiva longitud que permitan identificar si una serie presenta un comportamiento aleatorio. Para ello se realizan múltiples réplicas de longitud equivalente a la serie de precios $(L=261)$ con muestras generadas con comportamientos aleatorios (siguiendo caminatas aleatorias); dichas series son evaluadas con diferentes tamaños de subseries, hasta hallar aquella que estima un coeficiente de Hurst $H=0.5$, lo anterior mediante una prueba de intervalo de confianza.

Como resultados del ejercicio de calibración se reformula la hipótesis para que esta tenga en cuenta el intervalo de confianza identificado con una confianza de 0.95: la serie presenta un 
comportamiento aleatorio tipo caminata aleatoria, es decir: $H_{0}^{6}: 0.4804 \leq$ $H \leq 0.5245 ; H_{1}^{6}$ : Caso contrario. Para el presente trabajo es programada una función en el lenguaje R, la cual estima el coeficiente de Hurst (Anexo 1)

\section{Evaluación de la causalidad}

\section{entre productos para las distintas centrales de abastos}

A partir de los diversos métodos aplicados para identificar aleatoriedad en las series es posible estructurar modelos autorregresivos estacionarios; sin embargo, estos solo se enfocan en un producto a la vez. Teniendo presente el objetivo de evaluar la relación entre las diferentes centrales de abastos, se procede a determinar si existe una relación entre el precio de un producto y los mercados donde es comercializado, con base en los resultados de la etapa anterior. Para ello se aplica la prueba de Granger (1986), ya que a partir de esta es posible identificar si la precedencia de un precio en una serie, generado en un mercado específico, puede causar o potencialmente predecir cambios en un futuro precio de otra serie (del mismo producto en otra CA).

Para la aplicación de la metodología se realizan tres pasos básicos: 1) seleccionar series estacionarias y que tengan presencia en todas las centrales de abastos, 2) evaluar el efecto de rezagos de cada par de series (mismo producto para cada par de CA) y 3) identificar relaciones entre los mercados. El efecto de los rezagos y la causalidad se puede expresar mediante las siguientes ecuaciones:

$$
\begin{aligned}
& P_{t}^{1}=\sum_{(i=1)}^{n} \alpha_{i} P_{(t-i)}^{1}+\sum_{(j=1)}^{n} \beta_{j} P_{(t-j)}^{2}+u_{1 t} \\
& P_{t}^{2}=\sum_{(i=1)}^{n} \alpha_{i} P_{(t-i)}^{2}+\sum_{(=1)}^{n} \beta_{j} P_{(t-j)}^{1}+u_{2 t}
\end{aligned}
$$

Donde cada una de las series presentadas indica que el valor en un instante $t$ es expresado por sus valores anteriores, es decir, cada valor es explicado mediante un modelo autorregresivo en conjunto con el modelo regresivo desfasado de otra serie. La idea general es determinar si el valor de cada coeficiente $\alpha_{\mathrm{i}} \beta_{\mathrm{j}} \gamma_{\mathrm{i}}$ y $\delta_{\mathrm{j}}$ es significativo. A partir de la prueba propuesta por Gardner es posible encontrar cuatro escenarios:

- Que exista causalidad unidireccional del producto uno al producto dos.

- Que exista causalidad unidireccional del producto dos al producto uno.

- Que exista causalidad bidireccional.

- Que no exista causalidad.

Como hipótesis nula se evalúa la probabilidad de que el coeficiente de una serie perteneciente a otro mercado sea significativo $\mathrm{y}$, por tanto, se presenta una 
causalidad: $H_{0}^{7}: P\left(F_{\text {experimental }}>F_{\text {critico }}\right)$, $\mathrm{H}_{1}^{7}$ caso contario.

Ahora bien, con el propósito de determinar el número de rezagos dentro del modelo de regresión, se propone de manera iterativa para cada par de series por analizar, calcular los distintos $\mathrm{p}$-valores de las pruebas $\mathrm{F}$, desde uno hasta 25 rezagos (el equivalente en semanas de medio año). Después se selecciona el número de rezagos más significativos y sobre este número se determina si existe causalidad o no.

En último término se realiza un grafo con el propósito de representar las relaciones entre los mercados, para ello se propone simbolizar cada central de abasto como un nodo y mediante flechas indicar la cantidad de productos que cumplen la hipótesis de causalidad desde ese mercado hacia otro.

\section{RESULTADOS}

\section{Resultados del análisis de aleatoriedad}

Una vez aplicadas las diferentes pruebas, los p-valores son registrados en el Anexo 2 y, al mismo tiempo, se construye una tabla en la cual se resume la cantidad de veces que no es posible (con una confianza del 0.95) rechazar cada una de las hipótesis nulas (Tabla $2)$. Donde las filas indican las veces en que cada una de las seis hipótesis nulas no pudo ser rechazada, para un total máximo de 28 veces equivalentes a las series de productos agrícolas analizados por cada prueba en cada una de las centrales de abastos.

Tabla 2. Cantidad de productos a los que no es posible rechazar la hipótesis de aleatoriedad

\begin{tabular}{lcccccc}
\hline & Barranquilla & Bogotá & Bucaramanga & Manizales & Medellín & Villavicencio \\
\hline $\boldsymbol{H}_{\boldsymbol{0}}{ }^{\boldsymbol{1}}$ & 27 & 26 & 26 & 27 & 27 & 26 \\
$\boldsymbol{H}_{\boldsymbol{0}}{ }^{\boldsymbol{}}$ & 28 & 27 & 28 & 28 & 28 & 27 \\
$\boldsymbol{H}_{\boldsymbol{0}}{ }^{3}$ & 9 & 4 & 7 & 9 & 8 & 8 \\
$\boldsymbol{H}_{\boldsymbol{0}}{ }^{4}$ & 5 & 7 & 9 & 6 & 14 & 11 \\
$\boldsymbol{H}_{\boldsymbol{0}}$ & 28 & 28 & 28 & 28 & 28 & 28 \\
$\boldsymbol{H}_{\boldsymbol{0}}{ }^{\boldsymbol{}}$ & 0 & 1 & 0 & 1 & 4 & 1 \\
\hline
\end{tabular}

De manera general, de los resultados consignados en el Anexo 2 y la Tabla 2 , se puede concluir que no todas las centrales de abastos tienen la misma eficiencia para todos sus productos. Un ejemplo de lo anterior está en la serie de precios del arroz de primera (Producto P17), el cual en los mercados de 
Bogotá y Villavicencio no presenta un comportamiento de raíz unitaria (en las tres hipótesis de raíces unitarias), mientras que en Medellín, Manizales y Barranquilla la serie se comporta como una caminata aleatoria y, para el caso de la central de Bucaramanga, la serie presenta un comportamiento de raíz unitaria con deriva y sin tendencia. Ahora bien, con el propósito de determinar si existen tendencias en todos los mercados, todos los productos son analizados para todas las centrales de abastos en la aceptación de sus diferentes hipótesis: raíz unitaria con deriva y con tendencia (Tabla 3), raíz unitaria con deriva y sin tendencia (Tabla 4), raíz unitaria sin deriva y sin tendencia (Tabla 5), prueba de rachas (Tabla 6), análisis de autocorrelación (Tabla 7) y coeficiente de Hurst (Tabla 8).

De la Tabla 3 es posible determinar que solo para dos productos (manzana roja importada y uva red globe nacional) se encuentra un comportamiento de caminata aleatoria en las seis centrales de abastos, posiblemente por ser productos importados. Sin embargo, al analizar la variante denominada raíz unitaria con deriva y sin tendencia (Tabla 4), se encuentra que la mayoría de los productos $(96.43 \%)$ presenta dicho comportamiento en todas las centrales de abastos, con la salvedad del arroz de primera (P17), para el cual no es posible aceptar dicha hipótesis en los mercados de abastos de Bogotá y Villavicencio.

Respecto al proceso de raíz unitaria sin deriva y sin tendencia (Tabla 5), solo dos productos (manzana roja importada P12, manzana verde importada P13) presentan dicho comportamiento en todas las centrales de abastos. Ahora bien, para el caso del producto P13, de manera dual acepta dos tipos de hipótesis (caminata aleatoria y caminata aleatoria sin deriva y sin tendencia), lo cual es contradictorio $\mathrm{y}$, según explican DeJong, Nankervis, Savin y Whiteman (1992), puede ser debido a que la prueba de raíz unitaria puede presentar problemas para determinar la aleatoriedad de una serie, incluso cuando no existe autocorrelación.

Para el caso de la prueba de rachas, solo en tres productos no es posible aceptar la aleatoriedad en las seis centrales de abastos: pimentón (P9), espinaca (P19) y alverja verde en vaina (P26). Ahora bien, es necesario resaltar que debido a la naturaleza de la toma de datos (precio promedio de venta durante la semana), las series transformadas no eran dicotómicas, ya que en múltiples semanas de manera consecutiva se presentaba un precio promedio, por tanto, el algoritmo propuesto por Caeiro y Mateus (2014) reduce la longitud de las series para analizar, considerando solo aquellas en las que el precio cambia, 
lo cual puede generar distorsión en los resultados de la prueba. Respecto al análisis de autocorrelación mediante la prueba de Dubrin-Watson, se encuentra que ninguna serie obtiene p-valores superiores a 0.05 , por tanto, no es posible rechazar la hipótesis nula y las series estudiadas no presentan autocorrelación.

La prueba final indica que una vez estandarizadas las series de precios mediante su desviación estándar y teniendo en cuenta la autocorrelación propuesta por Lo (1991), en la mayoría de los productos no es posible aceptar la hipótesis de caminata aleatoria para la mayoría de las relaciones (producto-central de abastos), con la salvedad del brócoli, manzana roja importada, manzana verde importada y arveja verde en vaina (Medellín), remolacha (Manizales), papa criolla limpia (Bogotá) y alverja verde en vaina (Villavicencio). Los productos que aceptan la hipótesis nula de aleatoriedad según cada prueba son registrados en el diagrama de frecuencias de la Figura 1, donde el eje horizontal indica la cantidad de centrales en las que no se puede rechazar la hipótesis, el eje vertical, el número de veces que la prueba no es rechazada y el color indica la prueba.

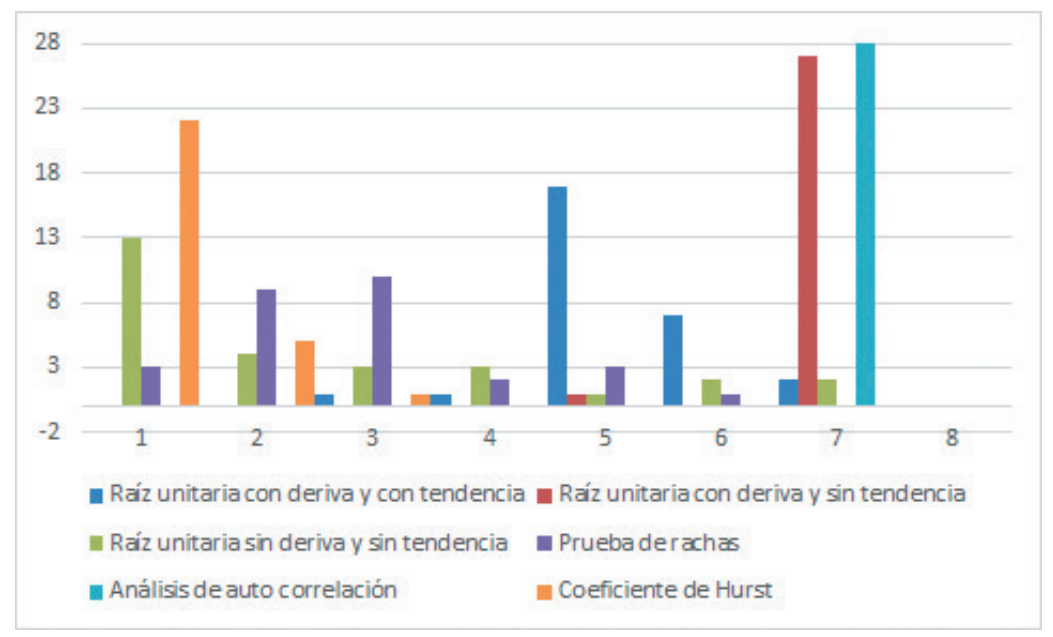

Figura 1. Diagrama de frecuencia de la cantidad pruebas no rechazadas en las seis centrales de abastos 
Evaluación de la hipótesis de eficiencia débil y análisis de causalidad en las centrales de abastos de Colombia Leonardo Hernán Talero-Sarmiento - Henry Lamos-Díaz - Edwin Alberto Garavito-Hernández

Tabla 3. Productos que aceptan y rechazan la hipótesis nula $H_{0}{ }^{1}$

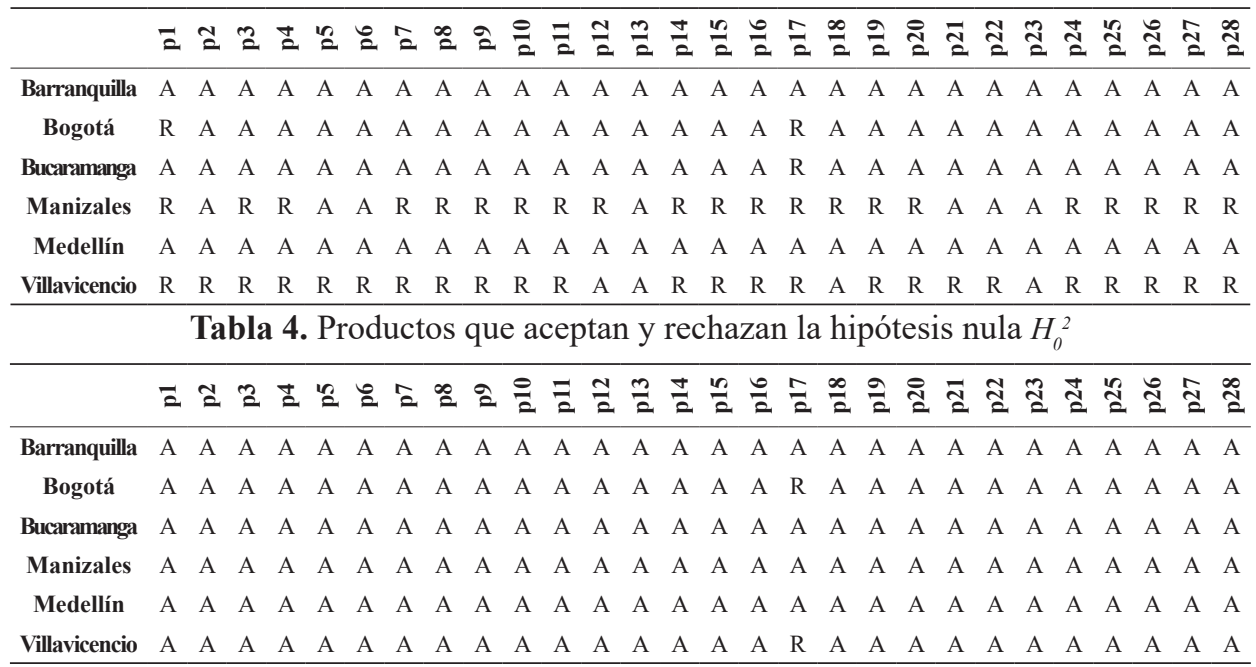

Tabla 5. Productos que aceptan y rechazan la hipótesis nula $H_{0}^{3}$

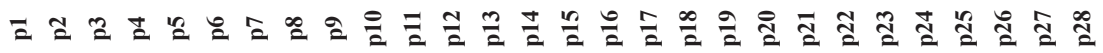

\begin{tabular}{lllllllllllllllllllllllllllllll}
\hline Barranquilla & $A$ & $A$ & $R$ & $A$ & $A$ & $R$ & $R$ & $R$ & $R$ & $R$ & $R$ & $A$ & $A$ & $R$ & $R$ & $R$ & $R$ & $R$ & $A$ & $R$ & $A$ & $R$ & $A$ & $R$ & $R$ & $R$ & $R$ & $R$
\end{tabular}

$\begin{array}{lllllllllllllllllllllllllllllllll}\text { Bogotá } & R & R & R & R & R & R & R & R & R & R & R & A & A & R & R & R & R & A & R & R & R & R & A & R & R & R & R & R\end{array}$

$\begin{array}{llllllllllllllllllllllllllllllllllll}\text { Bucaramanga } & R & A & R & R & R & R & R & R & R & R & R & A & A & R & R & R & R & A & A & R & R & A & A & R & R & R & R & R\end{array}$

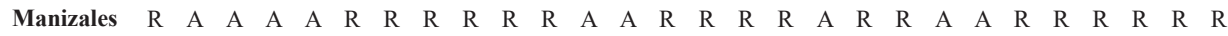

$\begin{array}{llllllllllllllllllllllllllllllll}\text { Medellín } & R & R & A & R & R & R & R & R & R & R & R & A & A & R & R & R & R & A & A & R & R & A & A & R & R & A & R & R\end{array}$

\begin{tabular}{llllllllllllllllllllllllllllllll} 
Villavicencio & $R$ & $R$ & $R$ & $R$ & $R$ & $A$ & $R$ & $R$ & $R$ & $R$ & $R$ & $A$ & $A$ & $R$ & $R$ & $R$ & $R$ & $A$ & $R$ & $R$ & $A$ & $A$ & $A$ & $R$ & $A$ & $R$ & $R$ & $R$ \\
\hline
\end{tabular}

Tabla 6. Productos que aceptan y rechazan la hipótesis nula $H_{0}^{4}$

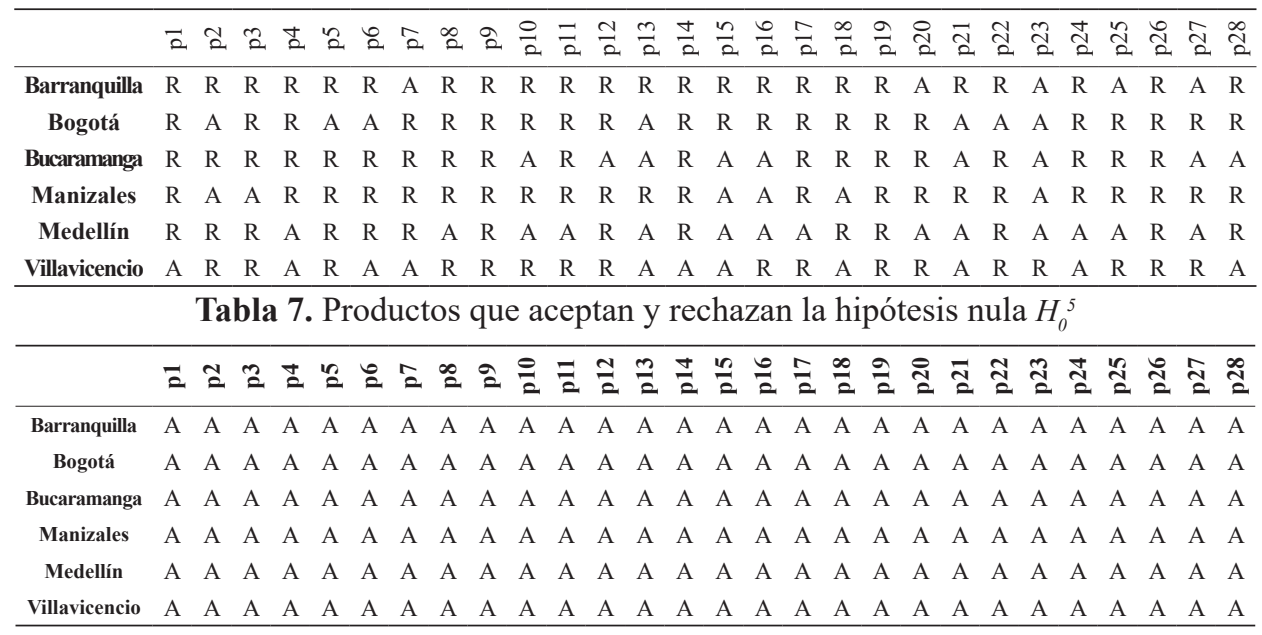


Tabla 8. Productos que aceptan y rechazan la hipótesis nula $H_{0}^{6}$

\begin{tabular}{|c|c|c|c|c|c|c|c|c|c|c|c|c|c|c|c|c|c|c|c|c|c|c|c|c|c|c|c|c|}
\hline & & 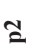 & 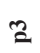 & 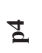 & 4 & 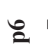 & & $x$ & & & & & & & & 2 & & & & ָั & $\overline{\widetilde{\Omega}}$ & こี & $\tilde{\check{\Sigma}}$ & & & & & \\
\hline & & & D & $\mathrm{R}$ & $\mathrm{R}$ & $\mathrm{R}$ & $\mathrm{R}$ & $\mathrm{R}$ & D & K & $\mathrm{K}$ & K & $\mathrm{R}$ & $\mathrm{R}$ & $\mathrm{R}$ & II & $\mathrm{K}$ & $\mathrm{R}$ & $\mathrm{K}$ & $\mathrm{R}$ & $\mathrm{R}$ & $\mathrm{R}$ & $\mathrm{R}$ & $\mathrm{R}$ & $\mathrm{R}$ & $\mathrm{R}$ & $\mathrm{R}$ & \\
\hline & $\mathrm{R}$ & II & II & $\mathrm{K}$ & $\mathrm{K}$ & $\mathrm{R}$ & $\mathrm{R}$ & $\mathrm{R}$ & IN & $\mathrm{K}$ & $\mathrm{K}$ & K & $\mathrm{R}$ & $\mathrm{R}$ & $\mathrm{R}$ & K & $\mathrm{R}$ & $\mathrm{R}$ & $\mathrm{R}$ & $\mathrm{R}$ & $\mathrm{R}$ & $\mathrm{R}$ & $\mathrm{R}$ & A & $\mathrm{R}$ & $\mathrm{R}$ & $\mathrm{R}$ & \\
\hline & $\mathrm{R}$ & $\mathrm{R}$ & $\mathrm{R}$ & $\mathrm{R}$ & $\mathrm{R}$ & $\mathrm{R}$ & $\mathrm{R}$ & $\mathrm{R}$ & $\mathrm{R}$ & $\mathrm{R}$ & $\mathrm{R}$ & $\mathrm{R}$ & $\mathrm{R}$ & $\mathrm{R}$ & $\mathrm{R}$ & $\mathrm{R}$ & $\mathrm{R}$ & $\mathrm{R}$ & $\mathrm{R}$ & $\mathrm{R}$ & $\mathrm{R}$ & $\mathrm{R}$ & $\mathrm{R}$ & $\mathrm{R}$ & $\mathrm{R}$ & $\mathrm{R}$ & $\mathrm{R}$ & \\
\hline & $\mathrm{R}$ & $\mathrm{R}$ & $\mathrm{R}$ & $\mathrm{R}$ & $\mathrm{R}$ & $\mathrm{R}$ & $\mathrm{R}$ & $\mathrm{R}$ & $\mathrm{R}$ & $\mathrm{R}$ & $\mathrm{R}$ & $\mathrm{R}$ & $\mathrm{R}$ & $\mathrm{R}$ & $\mathrm{R}$ & $\mathrm{R}$ & $\mathrm{R}$ & $\mathrm{R}$ & $\mathrm{R}$ & A & $\mathrm{R}$ & $\mathrm{R}$ & $\mathrm{R}$ & $\mathrm{R}$ & $\mathrm{R}$ & $\mathrm{R}$ & $\mathrm{R}$ & \\
\hline & $\mathrm{R}$ & A & $\mathrm{R}$ & $\mathrm{R}$ & $\mathrm{R}$ & $\mathrm{R}$ & $\mathrm{R}$ & $\mathrm{R}$ & $\mathrm{R}$ & $\mathrm{R}$ & $\mathrm{R}$ & A & A & $\mathrm{R}$ & $\mathrm{R}$ & $\mathrm{R}$ & $\mathrm{R}$ & $\mathrm{R}$ & $\mathrm{R}$ & $\mathrm{R}$ & $\mathrm{R}$ & $\mathrm{R}$ & $\mathrm{R}$ & $\mathrm{R}$ & $\mathrm{R}$ & A & $\mathrm{R}$ & \\
\hline Villavicencio & $\mathrm{R}$ & $\mathrm{R}$ & $\mathrm{R}$ & $\mathrm{R}$ & $\mathrm{R}$ & $\mathrm{R}$ & $\mathrm{R}$ & $\mathrm{R}$ & $\mathrm{R}$ & $\mathrm{R}$ & $\mathrm{R}$ & $\mathrm{R}$ & $\mathrm{R}$ & $\mathrm{R}$ & $\mathrm{R}$ & $\mathrm{R}$ & $\mathrm{R}$ & $\mathrm{R}$ & $\mathrm{R}$ & $\mathrm{R}$ & $\mathrm{R}$ & $\mathrm{R}$ & $\mathrm{R}$ & $\mathrm{R}$ & $\mathrm{R}$ & A & $\mathrm{R}$ & \\
\hline
\end{tabular}

\section{Resultados del análisis de causalidad}

Para el análisis son escogidos todos los productos que presentan una raíz unitaria con deriva y sin tendencia, debido a que es el patrón más común en todos los mercados y de esta manera solo se excluye del análisis el producto arroz de primera (P17). Los resultados de la cantidad de rezagos por considerar y los valores obtenidos de la prueba de causalidad se registran en los anexos 3 y 4 .

De las 810 pruebas aplicadas (cada relación entre los 27 productos y la permutación de centrales de abastos 6P3), se encuentra que no es posible refutar la hipótesis de causalidad en 294 casos (36.30\%), además, en el $78.91 \%$ de las causalidades, estas se presentan con un único rezago, $13.27 \%$ con dos rezagos, $5.10 \%$ con tres rezagos, 0.68 $\%$ con cuatro rezagos, $1.36 \%$ con cinco rezagos y $0.68 \%$ con ocho rezagos. Por otra parte, los productos en los que se confirma de manera más seguida la causalidad son: 20 ocasiones (de 30 posibles) para $\mathrm{P} 8$ (pepino cohombro) y 18 para P7 (lechuga batavia) y P16 (Tomate de árbol). Las veces que es aceptada la hipótesis de causalidad para cada producto se aprecian en la Figura 2.

\begin{tabular}{ccccccccc}
$P 1$ & $P 2$ & $P 3$ & $P 4$ & $P 5$ & $P 6$ & $P 7$ & $P 8$ & $P 9$ \\
\hline 11 & 11 & 2 & 12 & 8 & 6 & 18 & 20 & 13 \\
& & & & & & & & \\
$P 10$ & $P 11$ & $P 12$ & $P 13$ & $P 14$ & $P 15$ & $P 16$ & $P 17$ & $P 18$ \\
\hline 7 & 7 & 8 & 12 & 13 & 13 & 18 & 10 & 11 \\
$P 19$ & $P 20$ & $P 21$ & $P 22$ & $P 23$ & $P 24$ & $P 25$ & $P 26$ & $P 27$ \\
\hline 10 & 13 & 11 & 15 & 5 & 4 & 13 & 11 & 12
\end{tabular}

Figura 2. Frecuencia de la cantidad de pruebas no rechazadas para cada producto. 
Por otra parte, con el propósito de identificar las relaciones se genera el diagrama de la Figura 3, en el que son representadas mediante nodos las seis centrales de abastos, y su tamaño relativo indica la cantidad de veces que no es rechazada la hipótesis de causalidad desde su central hacia otra. Además, se representa la influencia de las centrales de abastos a partir de un vector que indica el número de productos que generan causalidad y la dirección de la misma: \{causalidad->;causalidad $<-;$ causalidad $<->\}$ : Barranquilla
$[49,19,23]$, Manizales [58,13,13], Villavicencio [34,30,19], Medellín $[19,35,12]$, Bogotá $[13,47,17]$ y Bucaramanga $[10,39,18]$ respectivamente. Teniendo en cuenta las interacciones dobles y los efectos unidireccionales, es posible inferir que el mercado de Manizales es el que mayor influencia tiene de los presentados en el estudio, mientras que el mercado de Bucaramanga es el que menos se ve afectado por los cambios en los otros.

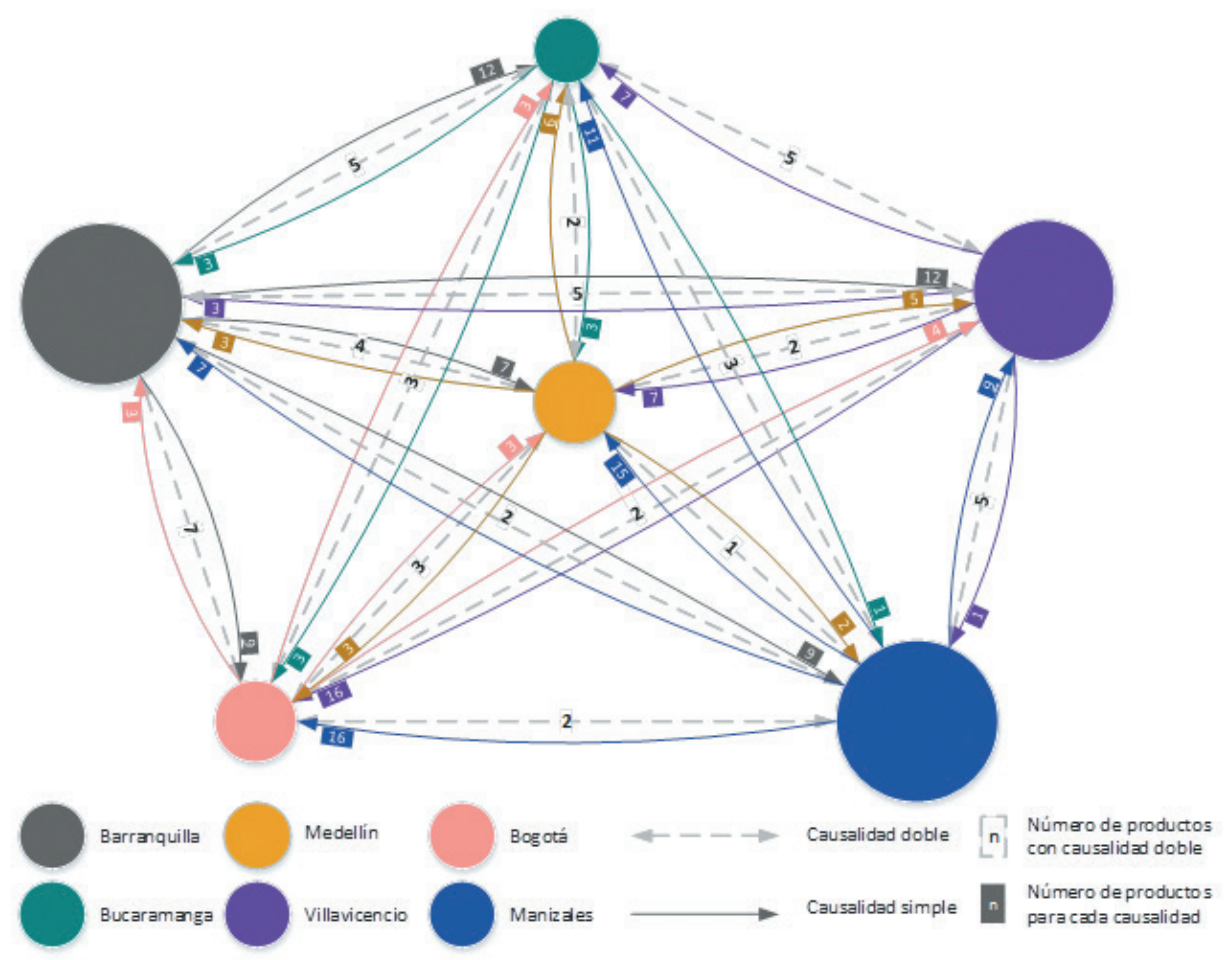

Figura 3. Diagrama de frecuencia de la cantidad de pruebas de causalidad no rechazadas en las seis centrales de abastos para los 27 productos. 


\section{DISCUSIÓN}

A partir de las pruebas aplicadas se concluye que no es posible rechazar la hipótesis de eficiencia de los mercados en todas las relaciones (producto/ central de abastos); no obstante, hay que tener en cuenta que los resultados de las pruebas aplicadas son no concluyentes en todas las relaciones, por lo cual se plantean dos posibles interpretaciones. La primera implica que según la dinámica de oferta y demanda de cada central de abastos, los productos pueden (o no) presentar comportamientos de aleatoriedad y, al ser diferente esa dinámica en cada central, dicho comportamiento no necesariamente debe ser igual. Por tanto, un futuro trabajo contemplado en este escenario es identificar si existen diferencias entre los precios de venta de los productos agrícolas comercializados en las centrales de abastos considerando dos grupos: aquellos en los cuales se acepta la hipótesis de eficiencia versus aquellos donde se rechaza $\mathrm{y}$, posteriormente, determinar si existe cointegración entre los mercados. Lo anterior, sumado a un análisis explicativo sobre la causalidad identificada, permitiría determinar oportunidades de inversión como la selección de la central de abastos en la cual se debe vender un producto específico o el efecto que tendría dicha central de abastos sobre el cambio de precio de un producto agrícola comercializado en otra central.
La segunda interpretación se enfoca en la exactitud de las pruebas. Por un lado, la prueba de raíz unitaria permite identificar la serie que presenta un comportamiento estocástico en sus diferentes versiones (considerando deriva o tendencia); sin embargo, durante el presente trabajo se rechaza la hipótesis en un $28.57 \%$ de las ocasiones, lo que indica que en la mayoría de las pruebas se determina que las series siguen un proceso no estacionario. Sin embargo, según DeJong et al. (1992), mediante la prueba aumentada de Dickey-Fuller se puede considerar en al menos un $50 \%$ de los casos el comportamiento no estacionario cuando el proceso es autorregresivo, es decir, la prueba aplicada tiende a aceptar el comportamiento no estacionario en más ocasiones de las garantizadas (Gujarati \& Porter, 2010), por tanto, para futuros trabajos se recomienda trabajar con variantes de la prueba de raíz unitaria como la propuesta por Bouri et al. (2017).

Por otra parte, la aplicación de la prueba de rachas fue realizada considerando una limitante, ya que al trabajar con el precio promedio semanal, el precio de ciertos productos se mantiene constante en el tiempo; lo cual, al tener en cuenta el comportamiento del algoritmo desarrollado por Caeiro y Mateus (2014), deriva en la transformación de la serie original a una más corta ignorando los periodos en los cuales 
no hay cambios (garantizando así una serie dicotómica). Por tanto, para futuros trabajos se recomienda estudiar el comportamiento de los precios de manera diaria con el propósito de plasmar las rachas inherentes de las series.

Respecto a la exactitud de la prueba $d$, el hecho de encontrar un valor $d$ significativo no es garantía de que no exista autocorrelación (Gujarati \& Porter, 2010), debido a que la prueba puede ser sensible a características como la longitud de la serie (con una tendencia a mejorar entre más datos existan) (Tillman, 1975). A pesar de ello, según los resultados se determina que para ninguna relación es posible refutar la hipótesis nula para un análisis semanal de los precios, lo cual para futuros trabajos puede ser contrastado con otra frecuencia de datos (particularmente, precios diarios).

Respecto a la última prueba, el coeficiente de Hurst se caracteriza por poseer una metodología relacionada más con la estimación que con el cálculo (Resta, 2012), lo que implica que mediante diversas metodologías pueden obtenerse valores distintos, además, que el nivel de memoria de la serie suele disminuir con la longitud de la misma (Peters, 2015). Durante el presente trabajo se aplica la variante propuesta por Andrew Lo (1991), la cual incorpora la autocorrelación para el reescalamiento de los datos, procurando así evidenciar relaciones de la serie con sus respectivos rezagos, aun cuando esta puede poseer un tamaño alto. Para futuros trabajos se recomienda hacer contrastes entre diferentes estimaciones del coeficiente de Hurst calibradas para longitudes específicas, con el fin de disminuir la incertidumbre sobre la efectividad o poder de la prueba.

En cuanto a las pruebas de causalidad, se recomienda para futuros trabajos llevar a cabo estudios enfocados en la dinámica de abastecimiento y flujos de productos en el marco de la logística entre centrales, con el propósito de determinar si la causalidad identificada se debe a que los productos son comercializados de una central a otra o, por otra parte, si elementos como cambios climáticos, políticos y demás, no tienen un efecto simultáneo en la geografía nacional.

\section{CONCLUSIONES}

Mediante el desarrollo metodológico propuesto para el presente trabajo se hace una extrapolación del concepto de mercado eficiente desde entorno bursátil al contexto de oferta y demanda de productos agrícolas en un centro de acopio. Además, a partir de los resultados parciales obtenidos fue posible hacer un análisis comparativo entre las centrales de abastos con el fin de determinar si el comportamiento de cada 
una es independiente de las otras. Esta propuesta, si bien se desvía de la aplicación teórica respecto a valores futuros (como en el caso de los commodities) y no contempla diversos elementos que afectan el mercado agrícola, tales como informalidad, concentración excesiva de tierra, falencias logísticas, ausencia de créditos y capacitación, entre otros, es un acercamiento novedoso con el cual se generan antecedentes empíricos en mercados donde se comercializa una gran cantidad de productos no transables. Esto ayuda para que en futuros trabajos se identifiquen oportunidades de inversión o comercialización (considerando los productos en cada CA), así como la formulación de políticas públicas que beneficien a los productores.

Por otra parte, respecto a las pruebas aplicadas se concluye que si bien la mayoría de los procesos históricos sugieren un comportamiento no estacionario, los resultados indican que existen diferencias entre las centrales de abastos y, por tanto, deben ser estudiadas como mercados diferentes; además, se concluye que los centros en Manizales, Barranquilla y Villavicencio influyen sobre los precios de los mercados de Bogotá, Bucaramanga y Medellín, por tanto, para futuros trabajos relacionados con la generación de precios y sus efectos es necesario no estudiar de manera independiente las centrales de abastos, sino incorporar los efectos de otras centrales.

\section{AGRADECIMIENTOS}

Los autores agradecen a la Vicerrectoría de Investigación y Extensión (VIE) de la Universidad Industrial de Santander (UIS), al programa de Maestría en Ingeniería Industrial y al grupo de investigación OPALO. También agradecen los comentarios y sugerencias de los evaluadores anónimos. Así mismo, asumen la responsabilidad por las opiniones contenidas en el presente artículo

\section{REFERENCIAS}

Bekkers, E., Brockmeier, M., Francois, J., \& Yang, F. (2017). Local Food Prices and International Price Transmission. World Development, 96, 216-230. https://doi. org/10.1016/j.worlddev.2017.03.008

Benavides, G. (2004). Price Volatility Forecasts for Agricultural Commodities: An Application of Historical Volatility Models, Option Implieds and Composite Approaches for Futures Prices of Corn and Wheat. SSRN Electronic Journal, 3(2), 40-59. https://doi.org/10.2139/ssrn.611062 
Evaluación de la hipótesis de eficiencia débil y análisis de causalidad en las centrales de abastos de Colombia Leonardo Hernán Talero-Sarmiento - Henry Lamos-Díaz - Edwin Alberto Garavito-Hernández

Bouri, E., Chang, T., \& Gupta, R. (2017). Testing the Efficiency of the Wine Market Using Unit Root Tests with Sharp and Smooth Breaks. Wine Economics and Policy, 6(2), 80-87. https://doi.org/10.1016/j.wep.2017.06.001

Caeiro, F. \& Mateus, A. (2014). Randtests: Testing Randomness in R. R Package Version, 1 .

Cárdenas, J. I. \& Vallejo, L. E. (2016). Agricultura y desarrollo rural en Colombia 2011-2013: una aproximación. Apuntes del Cenes, 35(62), 87. https://doi. org/10.19053/22565779.4411

Coronado, S., Ramírez, M. \& Celso, P. L. (2014). Inefficiency in the International Coffee Market: The Case of Colombian Arabica. African Journal of Agricultural Research, 9(5), 556-561.

DeJong, D. N., Nankervis, J. C., Savin, N. E. \& Whiteman, C. H. (1992). The Power Problems of Unit Root Test in Time Series with Autoregressive Errors. Journal of Econometrics, 53(1-3), 323-343. https://doi.org/10.1016/0304-4076(92)90090-E

Departamento Administrativo Nacional de Estadística -DANE-. (2017). Cuentas trimestrales, Colombia. Producto interno bruto (PIB) Boletín técnico cuarto trimestre de 2016. Bogotá: DANE.

Departamento Nacional de Planeación -DNP-. (2015). El campo colombiano: un camino hacia el bienestar y la paz. Misión para la transformación del campo. Recuperado de https://www.dnp.gov.co/programas/agricultura/Paginas/Informe-misi\%C3\%B3n-FInal.aspx.

Duarte-Duarte, J. B. \& Mascareñas Pérez-Iñigo, J. M. (2014a). Comprobación de la eficiencia débil en los principales mercados financieros latinoamericanos. Estudios Gerenciales, 30(133), 365-375. https://doi.org/10.1016/j.estger.2014.05.005

Duarte-Duarte, J. B. \& Mascareñas Pérez-Iñigo, J. M. M. (2014b). ¿Han sido los mercados bursátiles eficientes informacionalmente? Apuntes del Cenes, 33(57), 117. https://doi.org/10.19053/22565779.2906

Duarte-Duarte, J. B., Talero, L. H. \& Sierra, K. J. (2017). Evaluation of the Effect of Investor Psychology on an Artificial Stock Market Through its Degree of Efficiency. Contaduría y Administración, 62(4), 1361-1376. https://doi.org/10.1016/j. cya.2017.06.014

Durbin, J. \& Watson, G. S. (1951). Testing for Serial Correlation in Least Squares Regression. II. Biometrika, 38(1/2), 159. https://doi.org/10.2307/2332325

Fama, E. F. (1965). The Behavior of Stock-Market Prices. The Journal of Business, 38(1), 34. https://doi.org/10.1086/294743

Fama, E. F. (1991). Efficient Capital Markets: II. The Journal of Finance, 46(5), 15751617. https://doi.org/10.1111/j.1540-6261.1991.tb04636.x

Food and Agriculture Organization of the United Nations FAO. (2011). Food Price Volatility and the Right to Food. Rome: FAO. 
Geary, R. C. (1935). The Ratio of the Mean Deviation to the Standard Deviation as a Test of Normality. Biometrika, 27(3-4), 310-332. https://doi.org/10.1093/ biomet/27.3-4.310

Gómez, H. J. (2011). Política comercial y de competitividad del sector agrícola en Colombia. En Cuadernos Fedesarrollo (ed.), La política comercial del sector agrícola en Colombia (pp. 6-20). Bogotá: Cuadernos Fedesarrollo.

Granger, C. W. J. (1986). Developments in the Study of Cointegrated Economic Variables. Oxford Bulletin of Economics and Statistics, 48(3), 213-228. https://doi. org/10.1111/j.1468-0084.1986.mp48003002.x

Grossman, S. J. \& Stiglitz, J. E. (1980). On the Impossibility of Informationally Efficient Markets. The American Economic Review, 70(3), 393-408. Retrieved from www. jstor.org/stable/1805228.

Gujarati, D. N. \& Porter, D. C. (2010). Econometría. México: McGraw-Hill.

Hassan, A., Abdullah, M. S. \& Shah, Z. A. (2007). Testing of Random Walks and market efficiency in an emerging market: An empirical analysis of Karachi Stock Exchange. The Business Review, Cambridge, 9(1), 271-280.

Karali, B., \& Power, G. J. (2013). Short- and Long-Run Determinants of Commodity Price Volatility. American Journal of Agricultural Economics, 95(3), 724-738. https://doi.org/10.1093/ajae/aas122

Lachman, J. \& Jack, P. (2017). Study of Efficiency and Information Transmission for Agricultural Futures Markets: A Comparative Analysis between Buenos Aires and Chicago Using Monthly and Daily Data. Estudios Economicos, 34(69), 3-23.

Levy, R. A. (1967). The Theory of Random Walks: A Survey of Findings. The American Economist, 11(2), 34-48. https://doi.org/10.1177/056943456701100205

Lo, A. W. (1991). Long-Term Memory in Stock Market Prices. Econometrica, 59(5), 1279-1313. https://doi.org/10.2307/2938368

Lo, A. W. (2005). Reconciling Efficient Market with Behavioral Finance: The Adaptative Markets Hypothesis. The Journal of Investment Consulting, 7(2), 1-25. https:// doi.org/10.2139/ssrn.728864

Malkiel, B. G. (2003). The Efficient Market Hypothesis and Its Critics. Journal of Economic Perspectives, 17(1), 59-82. https://doi.org/10.1257/089533003321164958

Minot, N. (2014). Food Price Volatility in Sub-Saharan Africa: Has it Really Increased? Food Policy, 45, 45-56. https://doi.org/10.1016/j.foodpol.2013.12.008

Nisar, S. \& Hanif, M. (2012). Testing Weak Form of Efficient Market Hypothesis: Empirical Evidence from South-Asia. World Applied Sciences Journal, 17(4), 414-427. https://doi.org/10.3968/5524

Peters, E. E. (2015). Fractal Market Analysis: Applying Chaos Theory to Investment and Economics. Wiley Finance Editions. Retrieved from https://books.google.com.co/ 
Evaluación de la hipótesis de eficiencia débil y análisis de causalidad en las centrales de abastos de Colombia Leonardo Hernán Talero-Sarmiento - Henry Lamos-Díaz - Edwin Alberto Garavito-Hernández

books?hl=es\&lr=\&id=_bkoySKyc_cC\&oi=fnd\&pg=PA3\&dq=(Fractal+Marke$\mathrm{t}+$ Analysis:+Applying + Chaos + Theory+to+Investment+and+Economics\&ots $=\mathrm{s}-$ NzltsZ0IO\&sig=cnQpI_4F5zlD5blbhNH9omRATmo\%5Cnhttp://www.loc.gov/ catdir/description/wiley037/93028

Pfaff, B. (2008). Analysis of Integrated Series with R and Cointegrated Time. Use R! New York: Springer.

Resta, M. (2012). Hurst Exponent and its Applications in Time-series Analysis. Recent Patents on Computer Science, 5(3), 211-219. https://doi. org/10.2174/2213275911205030211

Ruiz-Porras, A. \& Ruiz-Robles, B. (2015). La hipótesis de eficiencia y la modelación de series bursátiles mexicanas: un análisis multivariado. Economía Informa, 390, 28-57. https://doi.org/10.1016/S0185-0849(15)30003-7

Savin, N. E. \& White, K. J. (1977). The Durbin-Watson Test for Serial Correlation with Extreme Sample Sizes or Many Regressors. Econometrica, 45(8), 1989. https:// doi.org/10.2307/1914122

Talero, L. H., Duarte, J. B. \& Garcés, L. D. (2017). La complejidad del mercado bursátil latinoamericano a partir de un modelo autómata celular conductual. Apuntes del Cenes, 36(64), 199-223. https://doi.org/10.19053/01203053.v36.n64.2017.5421

Tansuchat, R., Chang, C. \& McAleer, M. (2009). Modelling Long Memory Volatility in Agricultural Commodity Futures Returns. https://doi.org/10.2139/ssrn.1491890

Tejeda, H. A. \& Goodwin, B. K. (2009). Price Volatility, Nonlinearity, and Asymmetric Adjustments in Corn, Soybean, and Cattle Markets: Implications of Ethanol-Driven (Market) Shocks. In 2009 Conference, April 20-21, 2009, St. Louis, Missouri.

Tillman, J. A. (1975). The Power of the Durbin-Watson Test. Econometrica, 43(5/6), 959. https://doi.org/10.2307/1911337

Toth, A., Rendall, S., \& Reitsma, F. (2016). Resilient Food Systems: A Qualitative Tool for Measuring Food Resilience. Urban Ecosystems, 19(1), 19-43. https://doi. org/10.1007/s11252-015-0489-x

Velásquez, J. D. \& Aldana, M. A. (2007). Modelado del precio del café colombiano en la bolsa de Nueva York usando redes neuronales artificiales. Recuperado de http:// www.scielo.org.co/pdf/rfnam/v60n2/a16v60n2.pdf

Zeileis, A., \& Hothorn, T. (2002). Diagnostic Checking in Regression Relationships. $R$. News, 2(3), 7-10. 


\section{ANEXOS}

\section{Código de estimación del coeficiente programado en $\mathbf{R}$}

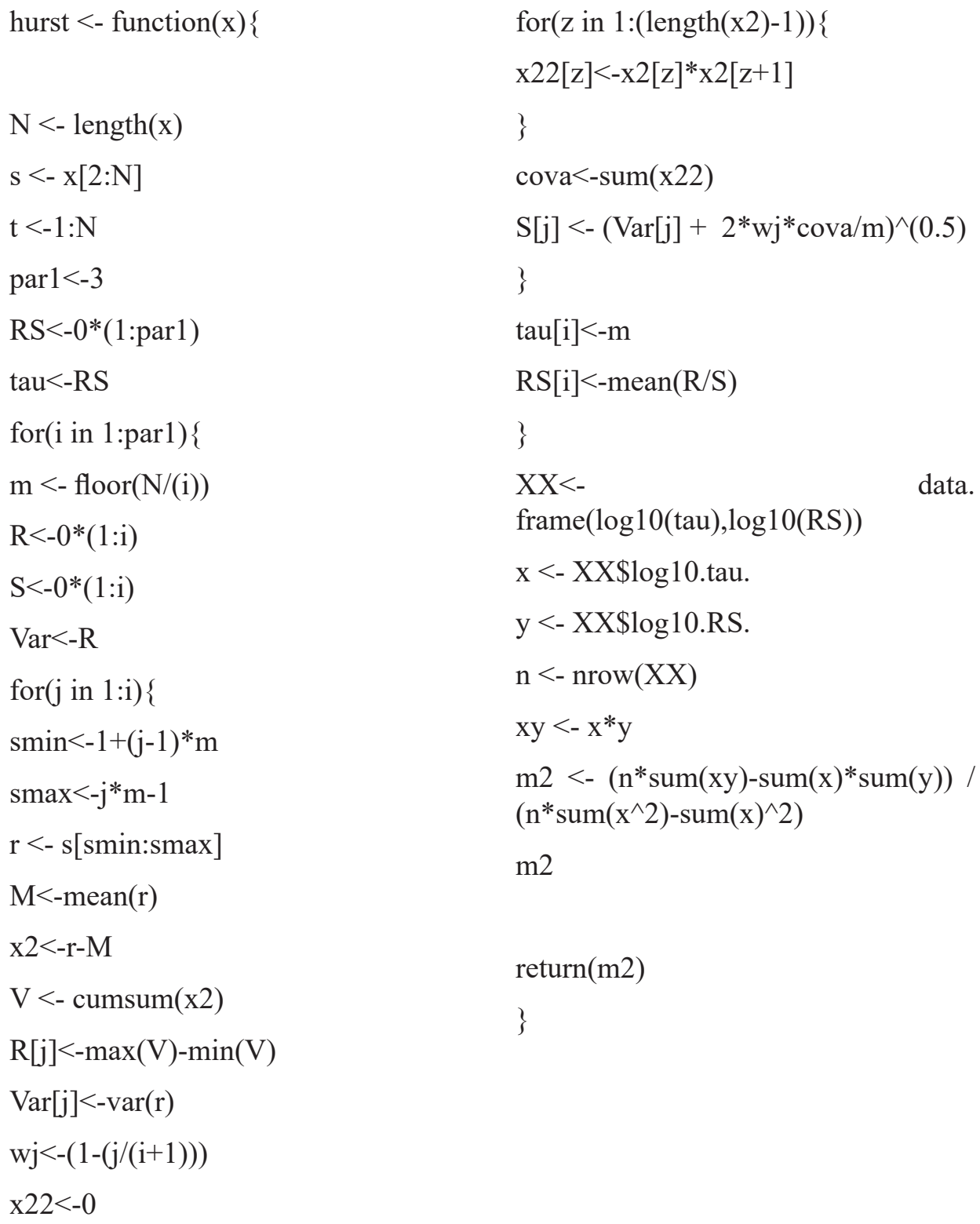


Evaluación de la hipótesis de eficiencia débil y análisis de causalidad en las centrales de abastos de Colombia Leonardo Hernán Talero-Sarmiento - Henry Lamos-Díaz - Edwin Alberto Garavito-Hernández

\section{P-valores obtenidos de las seis hipótesis para cada producto en las seis centrales de abastos}

\begin{tabular}{|c|c|c|c|c|c|c|c|c|c|c|c|c|c|c|c|c|c|c|}
\hline & \multicolumn{6}{|c|}{ Bogotá } & \multicolumn{6}{|c|}{ Bucaramanga } & \multicolumn{6}{|c|}{ Villavicencio } \\
\hline & $H_{0}^{1}$ & $H_{0}^{2}$ & $H_{0}^{3}$ & $H_{0}^{4}$ & $H_{0}^{5}$ & $H_{0}^{6}$ & $H_{0}^{1}$ & $H_{0}^{2}$ & $H_{0}^{3}$ & $H_{0}^{4}$ & $H_{0}^{5}$ & $H_{0}^{6}$ & $H_{0}^{1}$ & $H_{0}^{2}$ & $H_{0}^{2}$ & $H_{0}^{4}$ & $H_{0}^{5}$ & $H_{0}^{6}$ \\
\hline p1 & 0,06 & 0,00 & 0,67 & 0,90 & 0,00 & 0,24 & 0,01 & 0,00 & 0,51 & 0,22 & 0,00 & 0,18 & 0,01 & 0,00 & 0,37 & 0,02 & 0,00 & 0,30 \\
\hline p2 & 0,00 & 0,00 & 0,23 & 0,01 & 0,00 & 0,14 & 0,00 & 0,00 & 0,03 & 0,46 & 0,00 & 0,36 & 0,00 & 0,00 & 0,47 & 0,25 & 0,00 & 0,31 \\
\hline p3 & 0,04 & 0,00 & 0,34 & 0,14 & 0,00 & 0,18 & 0,00 & 0,00 & 0,78 & 0,90 & 0,00 & 0,17 & 0,03 & 0,00 & 0,42 & 0,38 & 0,00 & 0,24 \\
\hline $\mathrm{p} 4$ & 0,00 & 0,00 & 0,20 & 0,71 & 0,00 & 0,00 & 0,00 & 0,00 & 0,18 & 0,75 & 0,00 & 0,27 & 0,00 & 0,00 & 0,08 & 0,01 & 0,00 & 0,28 \\
\hline p5 & 0,00 & 0,00 & 0,06 & 0,03 & 0,00 & 0,60 & 0,00 & 0,00 & 0,05 & 0,84 & 0,00 & 0,37 & 0,00 & 0,00 & 0,07 & 0,80 & 0,00 & 0,23 \\
\hline p6 & 0,00 & 0,00 & 0,06 & 0,00 & 0,00 & 0,07 & 0,00 & 0,00 & 0,48 & 0,71 & 0,00 & 0,15 & 0,00 & 0,00 & 0,04 & 0,01 & 0,00 & 0,07 \\
\hline p7 & 0,02 & 0,00 & 0,19 & 0,46 & 0,00 & 0,33 & 0,00 & 0,00 & 0,48 & 0,08 & 0,00 & 0,30 & 0,00 & 0,00 & 0,55 & 0,03 & 0,00 & 0,32 \\
\hline p8 & 0,00 & 0,00 & 0,06 & 0,08 & 0,00 & 0,15 & 0,00 & 0,00 & 0,14 & 0,71 & 0,00 & 0,19 & 0,00 & 0,00 & 0,11 & 0,62 & 0,00 & 0,46 \\
\hline p9 & 0,00 & 0,00 & 0,11 & 0,71 & 0,00 & 0,42 & 0,00 & 0,00 & 0,07 & 0,11 & 0,00 & 0,32 & 0,00 & 0,00 & 0,31 & 0,71 & 0,00 & 0,39 \\
\hline p10 & 0,00 & 0,00 & 0,90 & 0,26 & 0,00 & 0,04 & 0,01 & 0,00 & 0,31 & 0,00 & 0,00 & 0,29 & 0,00 & 0,00 & 0,34 & 0,26 & 0,00 & 0,08 \\
\hline p11 & 0,00 & 0,00 & 0,77 & 0,90 & 0,00 & 0,35 & 0,00 & 0,00 & 0,72 & 0,90 & 0,00 & 0,47 & 0,00 & 0,00 & 0,79 & 0,71 & 0,00 & 0,46 \\
\hline p12 & 0,00 & 0,00 & 0,00 & 0,90 & 0,00 & 0,24 & 0,00 & 0,00 & 0,01 & 0,01 & 0,00 & 0,34 & 0,00 & 0,00 & 0,00 & 0,78 & 0,00 & 0,34 \\
\hline p13 & 0,00 & 0,00 & 0,00 & 0,00 & 0,00 & 0,81 & 0,00 & 0,00 & 0,02 & 0,00 & 0,00 & 0,20 & 0,01 & 0,00 & 0,03 & 0,00 & 0,00 & 0,35 \\
\hline p14 & 0,00 & 0,00 & 0,44 & 0,08 & 0,00 & 0,31 & 0,00 & 0,00 & 0,64 & 0,46 & 0,00 & 0,19 & 0,00 & 0,00 & 0,74 & 0,03 & 0,00 & 0,27 \\
\hline p15 & 0,00 & 0,00 & 0,24 & 0,21 & 0,00 & 0,24 & 0,00 & 0,00 & 0,64 & 0,00 & 0,00 & 0,39 & 0,00 & 0,00 & 0,60 & 0,01 & 0,00 & 0,17 \\
\hline p16 & 0,00 & 0,00 & 0,28 & 0,80 & 0,00 & 0,18 & 0,00 & 0,00 & 0,58 & 0,00 & 0,00 & 0,12 & 0,00 & 0,00 & 0,97 & 0,17 & 0,00 & 0,03 \\
\hline p17 & 0,15 & 0,13 & 0,60 & 0,05 & 0,00 & 0,45 & 0,06 & 0,04 & 0,16 & 0,97 & 0,00 & 0,10 & 0,08 & 0,06 & 0,24 & 0,30 & 0,00 & 0,20 \\
\hline p18 & 0,03 & 0,00 & 0,01 & 0,05 & 0,00 & 0,53 & 0,04 & 0,01 & 0,04 & 0,73 & 0,00 & 0,58 & 0,05 & 0,00 & 0,01 & 0,03 & 0,00 & 0,56 \\
\hline p19 & 0,01 & 0,00 & 0,23 & 0,26 & 0,00 & 0,40 & 0,00 & 0,00 & 0,02 & 0,41 & 0,00 & 0,61 & 0,00 & 0,00 & 0,11 & 0,38 & 0,00 & 0,44 \\
\hline p20 & 0,04 & 0,00 & 0,27 & 0,71 & 0,00 & 0,32 & 0,02 & 0,00 & 0,37 & 0,37 & 0,00 & 0,35 & 0,02 & 0,00 & 0,28 & 0,53 & 0,00 & 0,42 \\
\hline p21 & 0,00 & 0,00 & 0,10 & 0,01 & 0,00 & 0,09 & 0,00 & 0,00 & 0,11 & 0,00 & 0,00 & 0,11 & 0,00 & 0,00 & 0,04 & 0,00 & 0,00 & 0,20 \\
\hline p22 & 0,00 & 0,00 & 0,14 & 0,01 & 0,00 & 0,03 & 0,00 & 0,00 & 0,00 & 0,45 & 0,00 & 0,17 & 0,00 & 0,00 & 0,00 & 0,32 & 0,00 & 0,19 \\
\hline p23 & 0,00 & 0,00 & 0,02 & 0,00 & 0,00 & 0,23 & 0,00 & 0,00 & 0,01 & 0,00 & 0,00 & 0,08 & 0,00 & 0,00 & 0,02 & 0,08 & 0,00 & 0,18 \\
\hline p24 & 0,02 & 0,00 & 0,68 & 1,00 & 0,00 & 0,50 & 0,05 & 0,01 & 0,57 & 0,32 & 0,00 & 0,35 & 0,02 & 0,00 & 0,84 & 0,05 & 0,00 & 0,41 \\
\hline p25 & 0,00 & 0,00 & 0,42 & 0,75 & 0,00 & 0,43 & 0,01 & 0,01 & 0,08 & 0,06 & 0,00 & 0,74 & 0,00 & 0,00 & 0,01 & 0,13 & 0,00 & 0,63 \\
\hline p26 & 0,02 & 0,00 & 0,29 & 0,11 & 0,00 & 0,27 & 0,02 & 0,00 & 0,23 & 0,38 & 0,00 & 0,27 & 0,01 & 0,00 & 0,10 & 0,11 & 0,00 & 0,52 \\
\hline p27 & 0,02 & 0,00 & 0,37 & 0,62 & 0,00 & 0,33 & 0,05 & 0,00 & 0,28 & 0,01 & 0,00 & 0,30 & 0,04 & 0,00 & 0,23 & 0,32 & 0,00 & 0,23 \\
\hline p28 & 0,01 & 0,00 & 0,32 & 0,23 & 0,00 & 0,56 & 0,00 & 0,00 & 0,61 & 0,05 & 0,00 & 0,33 & 0,01 & 0,00 & 0,56 & 0,00 & 0,00 & 0,38 \\
\hline
\end{tabular}




\begin{tabular}{|c|c|c|c|c|c|c|c|c|c|c|c|c|c|c|c|c|c|c|}
\hline & \multicolumn{6}{|c|}{ Medellín } & \multicolumn{6}{|c|}{ Manizalez } & \multicolumn{6}{|c|}{ Barranquilla } \\
\hline & $H_{0}^{1}$ & $H_{0}^{2}$ & $H_{0}^{3}$ & $H_{0}^{4}$ & $H_{0}^{5}$ & $H_{0}^{6}$ & $H_{0}^{1}$ & $H_{0}^{2}$ & $H_{0}^{3}$ & $H_{0}^{4}$ & $H_{0}^{5}$ & $H_{0}^{6}$ & $H_{0}^{1}$ & $H_{0}^{2}$ & $H_{0}^{2}$ & $H_{0}^{4}$ & $H_{0}^{5}$ & $H_{0}^{6}$ \\
\hline p1 & 0,00 & 0,00 & 0,37 & 0,11 & 0,00 & 0,22 & 0,01 & 0,00 & 0,33 & 0,62 & 0,00 & 0,22 & 0,00 & 0,00 & 0,00 & 0,20 & 0,00 & 0,41 \\
\hline p2 & 0,00 & 0,00 & 0,20 & 1,00 & 0,00 & 0,49 & 0,00 & 0,00 & 0,01 & 0,01 & 0,00 & 0,16 & 0,00 & 0,00 & 0,01 & 0,17 & 0,00 & 0,35 \\
\hline p3 & 0,00 & 0,00 & 0,01 & 0,58 & 0,00 & 0,27 & 0,00 & 0,00 & 0,00 & 0,02 & 0,00 & 0,70 & 0,00 & 0,00 & 0,07 & 0,21 & 0,00 & 0,27 \\
\hline p4 & 0,00 & 0,00 & 0,25 & 0,02 & 0,00 & 0,09 & 0,00 & 0,00 & 0,00 & 0,25 & 0,00 & 0,39 & 0,00 & 0,00 & 0,02 & 0,15 & 0,00 & 0,41 \\
\hline p5 & 0,00 & 0,00 & 0,14 & 1,00 & 0,00 & 0,40 & 0,00 & 0,00 & 0,00 & 0,08 & 0,00 & 0,23 & 0,00 & 0,00 & 0,02 & 0,22 & 0,00 & 0,34 \\
\hline p6 & 0,00 & 0,00 & 0,36 & 0,32 & 0,00 & 0,25 & 0,00 & 0,00 & 0,29 & 0,62 & 0,00 & 0,23 & 0,00 & 0,00 & 0,54 & 1,00 & 0,00 & 0,10 \\
\hline p7 & 0,00 & 0,00 & 0,40 & 0,53 & 0,00 & 0,27 & 0,00 & 0,00 & 0,24 & 0,11 & 0,00 & 0,25 & 0,05 & 0,00 & 0,45 & 0,00 & 0,00 & 0,29 \\
\hline p8 & 0,00 & 0,00 & 0,07 & 0,00 & 0,00 & 0,26 & 0,00 & 0,00 & 0,36 & 0,66 & 0,00 & 0,36 & 0,00 & 0,00 & 0,12 & 0,16 & 0,00 & 0,19 \\
\hline p9 & 0,05 & 0,00 & 0,06 & 0,06 & 0,00 & 0,19 & 0,00 & 0,00 & 0,10 & 0,38 & 0,00 & 0,36 & 0,00 & 0,00 & 0,12 & 0,75 & 0,00 & 0,27 \\
\hline p10 & 0,00 & 0,00 & 1,00 & 0,01 & 0,00 & 0,16 & 0,00 & 0,00 & 0,13 & 0,18 & 0,00 & 0,24 & 0,00 & 0,00 & 0,63 & 0,98 & 0,00 & 0,53 \\
\hline p11 & 0,00 & 0,00 & 0,47 & 0,02 & 0,00 & 0,59 & 0,00 & 0,00 & 0,21 & 0,37 & 0,00 & 0,39 & 0,01 & 0,01 & 0,53 & 0,57 & 0,00 & 0,80 \\
\hline p12 & 0,00 & 0,00 & 0,00 & 0,58 & 0,00 & 0,50 & 0,00 & 0,00 & 0,00 & 0,83 & 0,00 & 0,29 & 0,01 & 0,01 & 0,03 & 0,58 & 0,00 & 0,38 \\
\hline p13 & 0,00 & 0,00 & 0,00 & 0,01 & 0,00 & 0,50 & 0,00 & 0,00 & 0,00 & 0,11 & 0,00 & 0,47 & 0,00 & 0,00 & 0,00 & 0,43 & 0,00 & 0,34 \\
\hline p14 & 0,00 & 0,00 & 0,78 & 0,62 & 0,00 & 0,35 & 0,00 & 0,00 & 0,40 & 0,25 & 0,00 & 0,37 & 0,00 & 0,00 & 0,50 & 0,05 & 0,00 & 0,15 \\
\hline p15 & 0,00 & 0,00 & 0,07 & 0,00 & 0,00 & 0,28 & 0,00 & 0,00 & 0,35 & 0,00 & 0,00 & 0,13 & 0,00 & 0,00 & 0,19 & 0,20 & 0,00 & 0,44 \\
\hline p16 & 0,00 & 0,00 & 0,51 & 0,00 & 0,00 & 0,00 & 0,00 & 0,00 & 0,46 & 0,00 & 0,00 & 0,05 & 0,00 & 0,00 & 0,60 & 0,08 & 0,00 & 0,12 \\
\hline p17 & 0,03 & 0,02 & 0,21 & 0,00 & 0,00 & 0,47 & 0,02 & 0,01 & 0,14 & 0,13 & 0,00 & 0,47 & 0,03 & 0,04 & 0,49 & 0,51 & 0,00 & 0,38 \\
\hline p18 & 0,01 & 0,01 & 0,01 & 0,56 & 0,00 & 0,56 & 0,00 & 0,00 & 0,00 & 0,01 & 0,00 & 0,37 & 0,06 & 0,02 & 0,08 & 0,14 & 0,00 & 0,48 \\
\hline p19 & 0,00 & 0,00 & 0,02 & 0,59 & 0,00 & 0,04 & 0,00 & 0,00 & 0,83 & 0,21 & 0,00 & 0,44 & 0,00 & 0,00 & 0,03 & 0,35 & 0,00 & 0,36 \\
\hline p20 & 0,02 & 0,00 & 0,30 & 0,03 & 0,00 & 0,02 & 0,25 & 0,00 & 0,11 & 0,25 & 0,00 & 0,48 & 0,03 & 0,00 & 0,22 & 0,00 & 0,00 & 0,32 \\
\hline p21 & 0,00 & 0,00 & 0,18 & 0,03 & 0,00 & 0,24 & 0,00 & 0,00 & 0,01 & 0,15 & 0,00 & 0,38 & 0,00 & 0,00 & 0,00 & 0,49 & 0,00 & 0,79 \\
\hline p22 & 0,00 & 0,00 & 0,01 & 0,49 & 0,00 & 0,27 & 0,00 & 0,00 & 0,00 & 0,76 & 0,00 & 0,26 & 0,00 & 0,00 & 0,33 & 0,14 & 0,00 & 0,53 \\
\hline p23 & 0,01 & 0,00 & 0,02 & 0,00 & 0,00 & 0,06 & 0,00 & 0,00 & 0,11 & 0,00 & 0,00 & 0,10 & 0,00 & 0,00 & 0,02 & 0,00 & 0,00 & 0,36 \\
\hline p24 & 0,04 & 0,00 & 0,72 & 0,00 & 0,00 & 0,56 & 0,01 & 0,00 & 0,59 & 0,14 & 0,00 & 0,45 & 0,01 & 0,00 & 0,78 & 0,80 & 0,00 & 0,31 \\
\hline p25 & 0,00 & 0,00 & 0,13 & 0,03 & 0,00 & 0,68 & 0,00 & 0,00 & 0,64 & 0,24 & 0,00 & 0,70 & 0,00 & 0,00 & 0,16 & 0,02 & 0,00 & 0,58 \\
\hline p26 & 0,00 & 0,00 & 0,03 & 0,53 & 0,00 & 0,49 & 0,00 & 0,00 & 0,43 & 0,80 & 0,00 & 0,33 & 0,01 & 0,01 & 0,09 & 0,44 & 0,00 & 0,25 \\
\hline p27 & 0,02 & 0,00 & 0,46 & 0,01 & 0,00 & 0,14 & 0,02 & 0,00 & 0,18 & 0,32 & 0,00 & 0,20 & 0,04 & 0,00 & 0,26 & 0,05 & 0,00 & 0,30 \\
\hline p28 & 0,00 & 0,00 & 0,53 & 0,38 & 0,00 & 0,36 & 0,01 & 0,00 & 0,25 & 0,79 & 0,00 & 0,25 & 0,01 & 0,01 & 0,35 & 0,93 & 0,00 & 0,60 \\
\hline
\end{tabular}


Evaluación de la hipótesis de eficiencia débil y análisis de causalidad en las centrales de abastos de Colombia Leonardo Hernán Talero-Sarmiento - Henry Lamos-Díaz - Edwin Alberto Garavito-Hernández

3. Cantidad de desfases considerados para evaluar la hipótesis de causalidad para todos los productos en las seis centrales de abastos

\begin{tabular}{|c|c|c|c|c|c|c|c|c|c|c|c|c|c|c|c|}
\hline Desde & Hacia & P1 & P2 & P3 & P4 & P5 & P6 & P7 & P8 & P9 & P10 & P11 & P12 & P13 & P14 \\
\hline Bo/tá & $\mathrm{B} / \mathrm{ga}$ & 2 & 1 & 1 & 3 & 1 & 2 & 2 & 1 & 1 & 1 & 1 & 1 & 1 & 1 \\
\hline Bo/tá & Vi/cio & 2 & 3 & 1 & 5 & 1 & 1 & 2 & 1 & 1 & 1 & 1 & 1 & 1 & 1 \\
\hline Bo/tá & Me/lín & 1 & 1 & 1 & 1 & 2 & 3 & 1 & 1 & 1 & 3 & 1 & 1 & 3 & 1 \\
\hline Bo/tá & Ma/les & 2 & 1 & 1 & 1 & 2 & 3 & 2 & 1 & 1 & 1 & 1 & 1 & 3 & 1 \\
\hline Bo/tá & $\mathrm{Ba} / \mathrm{lla}$ & 1 & 1 & 1 & 1 & 1 & 3 & 1 & 1 & 1 & 1 & 1 & 1 & 1 & 1 \\
\hline $\mathrm{B} / \mathrm{ga}$ & Bo/tá & 1 & 2 & 1 & 4 & 1 & 1 & 1 & 2 & 2 & 1 & 1 & 1 & 2 & 3 \\
\hline $\mathrm{B} / \mathrm{ga}$ & Vi/cio & 1 & 2 & 1 & 4 & 1 & 1 & 1 & 1 & 1 & 1 & 1 & 1 & 1 & 1 \\
\hline $\mathrm{B} / \mathrm{ga}$ & Me/llín & 1 & 1 & 1 & 1 & 2 & 4 & 1 & 1 & 1 & 3 & 1 & 1 & 3 & 1 \\
\hline $\mathrm{B} / \mathrm{ga}$ & Ma/les & 1 & 1 & 1 & 2 & 1 & 1 & 1 & 2 & 1 & 1 & 1 & 2 & 3 & 1 \\
\hline $\mathrm{B} / \mathrm{ga}$ & $\mathrm{Ba} / \mathrm{lla}$ & 1 & 1 & 1 & 1 & 1 & 2 & 1 & 3 & 1 & 1 & 1 & 1 & 1 & 1 \\
\hline Vi/cio & Bo/tá & 1 & 2 & 1 & 5 & 1 & 1 & 1 & 1 & 2 & 2 & 1 & 1 & 2 & 1 \\
\hline Vi/cio & $\mathrm{B} / \mathrm{ga}$ & 2 & 1 & 1 & 5 & 1 & 2 & 2 & 1 & 1 & 1 & 1 & 1 & 1 & 1 \\
\hline Vi/cio & Me/llín & 1 & 1 & 1 & 1 & 2 & 3 & 1 & 1 & 1 & 3 & 1 & 1 & 3 & 1 \\
\hline Vi/cio & Ma/les & 1 & 2 & 1 & 1 & 2 & 3 & 2 & 1 & 1 & 1 & 1 & 1 & 3 & 1 \\
\hline Vi/cio & $\mathrm{Ba} / \mathrm{lla}$ & 1 & 1 & 1 & 1 & 1 & 3 & 1 & 1 & 1 & 2 & 1 & 1 & 1 & 1 \\
\hline Me/llín & Bo/tá & 1 & 2 & 1 & 5 & 1 & 1 & 1 & 1 & 2 & 1 & 1 & 1 & 8 & 2 \\
\hline Me/lín & $\mathrm{B} / \mathrm{ga}$ & 1 & 1 & 1 & 4 & 1 & 2 & 2 & 1 & 1 & 1 & 1 & 1 & 1 & 1 \\
\hline Me/llín & Vi/cio & 1 & 2 & 1 & 5 & 1 & 1 & 1 & 1 & 1 & 1 & 1 & 1 & 1 & 1 \\
\hline Me/llín & Ma/les & 1 & 3 & 1 & 1 & 1 & 1 & 1 & 1 & 1 & 1 & 3 & 1 & 3 & 2 \\
\hline Me/llín & $\mathrm{Ba} / \mathrm{lla}$ & 1 & 1 & 1 & 1 & 1 & 2 & 1 & 1 & 1 & 1 & 1 & 1 & 2 & 1 \\
\hline Ma/les & Bo/tá & 1 & 2 & 1 & 5 & 1 & 1 & 1 & 1 & 2 & 1 & 1 & 1 & 8 & 1 \\
\hline Ma/les & $\mathrm{B} / \mathrm{ga}$ & 1 & 1 & 1 & 5 & 1 & 3 & 2 & 1 & 1 & 1 & 1 & 1 & 1 & 1 \\
\hline Ma/les & Vi/cio & 1 & 2 & 1 & 5 & 1 & 4 & 1 & 1 & 1 & 2 & 1 & 1 & 1 & 2 \\
\hline Ma/les & Me/llín & 1 & 1 & 1 & 1 & 2 & 1 & 1 & 1 & 1 & 3 & 1 & 1 & 3 & 1 \\
\hline Ma/les & $\mathrm{Ba} / \mathrm{lla}$ & 1 & 1 & 1 & 1 & 1 & 3 & 1 & 1 & 1 & 1 & 1 & 1 & 2 & 1 \\
\hline $\mathrm{Ba} / \mathrm{lla}$ & Bo/tá & 1 & 2 & 1 & 5 & 1 & 1 & 1 & 2 & 2 & 1 & 1 & 1 & 2 & 2 \\
\hline $\mathrm{Ba} / \mathrm{lla}$ & $\mathrm{B} / \mathrm{ga}$ & 1 & 1 & 1 & 5 & 1 & 2 & 2 & 1 & 1 & 1 & 1 & 1 & 1 & 1 \\
\hline $\mathrm{Ba} / \mathrm{lla}$ & $\mathrm{Vi} /$ cio & 1 & 2 & 1 & 4 & 1 & 1 & 1 & 1 & 1 & 1 & 1 & 1 & 1 & 1 \\
\hline $\mathrm{Ba} / \mathrm{lla}$ & Me/llín & 1 & 3 & 1 & 1 & 2 & 4 & 1 & 1 & 1 & 1 & 1 & 1 & 3 & 1 \\
\hline $\mathrm{Ba} / \mathrm{lla}$ & Ma/les & 1 & 1 & 1 & 1 & 1 & 1 & 1 & 1 & 1 & 1 & 1 & 1 & 3 & 1 \\
\hline \multicolumn{16}{|c|}{$\begin{array}{c}\text { Ba/lla: Barranquilla, Bo/tá: Bogotá, B/ga: Bucaramanga, Ma/les: Manizales, } \\
\text { Me/llín: Medellín, Vi/cio: Villavicencio }\end{array}$} \\
\hline
\end{tabular}


Apuntes CENES Volumen 38, Número 67

enero - junio 2019. Págs. 35-69

\begin{tabular}{|c|c|c|c|c|c|c|c|c|c|c|c|c|c|c|}
\hline Desde & Hacia & P15 & P16 & P17 & P18 & P19 & P20 & P21 & P22 & P23 & P24 & P25 & P26 & P27 \\
\hline Bo/tá & $\mathrm{B} / \mathrm{ga}$ & 1 & 2 & 1 & 1 & 3 & 3 & 3 & 1 & 1 & 2 & 1 & 1 & 1 \\
\hline Bo/tá & Vi/cio & 1 & 2 & 1 & 1 & 2 & 1 & 1 & 1 & 1 & 1 & 1 & 1 & 1 \\
\hline Bo/tá & Me/lín & 1 & 2 & 1 & 1 & 1 & 1 & 1 & 1 & 1 & 1 & 1 & 1 & 1 \\
\hline Bo/tá & Ma/les & 1 & 2 & 2 & 1 & 3 & 1 & 2 & 1 & 1 & 1 & 1 & 1 & 1 \\
\hline Bo/tá & $\mathrm{Ba} / \mathrm{lla}$ & 1 & 2 & 2 & 1 & 3 & 4 & 1 & 1 & 4 & 1 & 2 & 3 & 1 \\
\hline $\mathrm{B} / \mathrm{ga}$ & Bo/tá & 1 & 1 & 3 & 1 & 1 & 1 & 1 & 1 & 1 & 2 & 1 & 1 & 2 \\
\hline $\mathrm{B} / \mathrm{ga}$ & Vi/cio & 1 & 1 & 1 & 1 & 1 & 1 & 1 & 1 & 1 & 1 & 2 & 1 & 1 \\
\hline $\mathrm{B} / \mathrm{ga}$ & Me/lín & 1 & 1 & 1 & 1 & 1 & 1 & 1 & 1 & 1 & 1 & 1 & 1 & 1 \\
\hline $\mathrm{B} / \mathrm{ga}$ & Ma/les & 1 & 2 & 2 & 1 & 1 & 1 & 2 & 1 & 1 & 1 & 1 & 1 & 1 \\
\hline B/ga & $\mathrm{Ba} / \mathrm{lla}$ & 1 & 2 & 2 & 1 & 1 & 4 & 1 & 1 & 1 & 1 & 3 & 3 & 1 \\
\hline Vi/cio & Bo/tá & 1 & 1 & 1 & 1 & 1 & 1 & 1 & 1 & 1 & 2 & 1 & 1 & 1 \\
\hline Vi/cio & $\mathrm{B} / \mathrm{ga}$ & 1 & 2 & 1 & 1 & 1 & 2 & 1 & 1 & 1 & 2 & 2 & 1 & 1 \\
\hline Vi/cio & Me/llín & 1 & 2 & 1 & 1 & 1 & 1 & 1 & 1 & 1 & 1 & 1 & 1 & 1 \\
\hline Vi/cio & Ma/les & 1 & 2 & 2 & 1 & 1 & 1 & 3 & 1 & 1 & 1 & 1 & 2 & 1 \\
\hline Vi/cio & $\mathrm{Ba} / \mathrm{lla}$ & 1 & 1 & 2 & 1 & 3 & 4 & 1 & 1 & 1 & 1 & 2 & 1 & 1 \\
\hline Me/lín & Bo/tá & 1 & 1 & 1 & 1 & 1 & 1 & 3 & 1 & 1 & 2 & 1 & 1 & 1 \\
\hline Me/llín & B/ga & 1 & 1 & 1 & 1 & 1 & 1 & 3 & 1 & 1 & 2 & 2 & 1 & 1 \\
\hline Me/lín & Vi/cio & 1 & 1 & 1 & 1 & 1 & 2 & 3 & 1 & 1 & 1 & 1 & 1 & 1 \\
\hline Me/lín & Ma/les & 1 & 2 & 2 & 1 & 1 & 1 & 2 & 1 & 1 & 3 & 1 & 1 & 1 \\
\hline Me/lín & $\mathrm{Ba} / \mathrm{lla}$ & 1 & 2 & 2 & 1 & 1 & 4 & 1 & 1 & 1 & 1 & 1 & 1 & 1 \\
\hline Ma/les & Bo/tá & 1 & 1 & 1 & 1 & 1 & 1 & 1 & 1 & 1 & 2 & 1 & 1 & 1 \\
\hline Ma/les & $\mathrm{B} / \mathrm{ga}$ & 1 & 1 & 1 & 1 & 1 & 1 & 1 & 1 & 1 & 2 & 1 & 1 & 1 \\
\hline Ma/les & Vi/cio & 1 & 1 & 1 & 1 & 1 & 1 & 1 & 1 & 1 & 1 & 1 & 1 & 1 \\
\hline Ma/les & Me/llín & 1 & 1 & 1 & 1 & 1 & 1 & 1 & 1 & 1 & 1 & 1 & 1 & 1 \\
\hline Ma/les & $\mathrm{Ba} / \mathrm{lla}$ & 1 & 1 & 2 & 1 & 1 & 13 & 1 & 1 & 1 & 1 & 1 & 1 & 1 \\
\hline $\mathrm{Ba} / \mathrm{lla}$ & Bo/tá & 1 & 1 & 3 & 1 & 1 & 1 & 1 & 1 & 1 & 2 & 1 & 1 & 1 \\
\hline $\mathrm{Ba} / \mathrm{lla}$ & $\mathrm{B} / \mathrm{ga}$ & 1 & 1 & 2 & 1 & 1 & 1 & 3 & 1 & 1 & 2 & 1 & 1 & 1 \\
\hline $\mathrm{Ba} / \mathrm{lla}$ & Vi/cio & 1 & 1 & 2 & 1 & 1 & 1 & 1 & 1 & 1 & 1 & 1 & 1 & 1 \\
\hline $\mathrm{Ba} / \mathrm{lla}$ & Me/lín & 1 & 1 & 1 & 1 & 1 & 1 & 1 & 1 & 1 & 1 & 1 & 1 & 1 \\
\hline $\mathrm{Ba} / \mathrm{lla}$ & Ma/les & 1 & 1 & 2 & 1 & 1 & 1 & 2 & 1 & 1 & 1 & 1 & 1 & 1 \\
\hline
\end{tabular}

Ba/lla: Barranquilla, Bo/tá: Bogotá, B/ga: Bucaramanga, Ma/les: Manizales, Me/llín: Medellín, Vi/cio: Villavicencio 
Evaluación de la hipótesis de eficiencia débil y análisis de causalidad en las centrales de abastos de Colombia Leonardo Hernán Talero-Sarmiento - Henry Lamos-Díaz - Edwin Alberto Garavito-Hernández

\section{P-valores obtenidos de la hipótesis de causalidad para todos los productos en las seis centrales de abastos}

\begin{tabular}{|c|c|c|c|c|c|c|c|c|c|c|c|c|c|c|c|}
\hline Desde & acia & 1 & P2 & P3 & P4 & P5 & P6 & P7 & P8 & P9 & P10 & P11 & P12 & P13 & P14 \\
\hline Bo/tá & & & & & 0,01 & & & & 0,00 & 0,01 & 0,88 & 0,39 & 0,12 & 0,01 & 0,00 \\
\hline Bo/tá & & 0,78 & & & 0,36 & 0,40 & & & 0,00 & 0,21 & 0,00 & 0,78 & 0,04 & 0,00 & 0,66 \\
\hline Bo/tá & e/lín &, 42 & 0,81 & 0,53 & 1,00 & 0,78 & 0,13 & 0,42 & 0,00 & 0,08 & 0,13 & 0,02 & 0,43 & 0,00 & 0,52 \\
\hline Bo/tá & lloc & 0,09 & 0,00 & 0,99 & 0,49 & 0,42 & 0,17 & 0,36 & 0,09 & 0,98 & 0,56 & 0,33 & 0,85 & 0,00 & 0,20 \\
\hline Bo/tá & & 0,71 & 0,00 & 0,49 & 0,04 & 0,04 & 0,89 & 0,99 & 0,00 & 0,00 & 0,69 & 0,07 & 0,05 & 0,11 & 0,23 \\
\hline $\mathrm{B} / \mathrm{ga}$ & & 0,11 & 0,71 & & 0,07 & 0,32 & 0,89 & & 0,04 & 0,02 & 0,01 & 0,03 & 0,37 & 0,32 & 0,25 \\
\hline B/ga & i/cio & 0,24 & 0,08 & 0,18 & 0,06 & 0,91 & 0,82 & 0,00 & 0,99 & 0,39 & 0,14 & 0,12 & 0,14 & 0,09 & 0,56 \\
\hline B/ga & e/llín & 0,63 & 0,10 & 0,33 & 0,39 & 0,15 & 0,62 & 0,02 & 0,41 & 0,20 & 0,15 & 0,13 & 0,90 & 0,36 & 0,88 \\
\hline $\mathrm{B} / \mathrm{ga}$ & Ma/les & 0,59 & 0,34 & 0,97 & 0,01 & 0,59 & 0,79 & 0,48 & 0,18 & 0,06 & 0,55 & 0,82 & 0,41 & 0,59 & 0,78 \\
\hline $\mathrm{B} / \mathrm{ga}$ & 3a/lla & 0,00 & 0,45 & 0,95 & 0,25 & 0,26 & 0,00 & 0,78 & 0,65 & 0,12 & 0,23 & 1,00 & 0,02 & 0,00 & 0,53 \\
\hline Vi/cio & o/tá & 0,00 & 0,00 & 0,00 & 0,00 & 0,00 & 0,02 & 0,00 & 0,00 & 0,28 & 0,12 & 0,03 & 0,31 & 0,72 & 0,00 \\
\hline Vi/cio & & 0,01 & & & 0,15 & 0,69 & & & 0,00 & & 0,83 & 0,31 & & 0,71 & 0,00 \\
\hline Vi/cio & 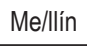 & 0,45 & 0,03 & 0,86 & 0,65 & 0,94 & 0,11 & 0,30 & 0,00 & 0,02 & 0,30 & 0,01 & 0,97 & 0,90 & 0,03 \\
\hline Vi/cio & Ma/les & 0,11 & 0,00 & 0,98 & 0,17 & 0,00 & 0,08 & 0,00 & 0,19 & 0,44 & 0,78 & 0,43 & 0,88 & 0,79 & 0,07 \\
\hline Vi/cio & $\mathrm{Ba} / \mathrm{lla}$ & 0,08 & 0,01 & 0,54 & 0,06 & 0,57 & 0,50 & 0,09 & 0,00 & 0,02 & 0,14 & 0,48 & 0,03 & 0,00 & 0,22 \\
\hline Me/llín & Bo/tá & 0,25 & 0,10 & 0,21 & 0,28 & 0,27 & 0,01 & 0,00 & 0,16 & 0,13 & 0,18 & 0,31 & 0,07 & 0,00 & 0,09 \\
\hline Me/lín & $\mathrm{B} / \mathrm{ga}$ & 0,20 & 0,39 & 0,76 & 0,00 & 0,35 & 0,14 & 0,03 & 0,00 & 0,39 & 0,38 & 0,82 & 0,52 & 0,75 & 0,02 \\
\hline Me/lín & & 0,01 & 0,92 & & 0,30 & 0,80 & 0,02 & 0,02 & 0,06 & 0,33 & 0,11 & 0,71 & 0,56 & 0,84 & 0,03 \\
\hline Me/llín & & & & & 0,28 & & & 0,67 & & & 0,39 & 0,78 & 0,08 & 0,54 & 0,33 \\
\hline Me/llín & $\mathrm{Ba} / \mathrm{lla}$ & 0,07 & 0,52 & 0,85 & 0,11 & 0,15 & 0,35 & 0,21 & 0,01 & 0,00 & 0,01 & 0,85 & 0,11 & 0,08 & 0,66 \\
\hline Ma/les & Bo/tá & 0,00 & 0,00 & 0,49 & 0,07 & 0,00 & 0,19 & 0,00 & 0,00 & 0,01 & 0,92 & 0,08 & 0,84 & 0,00 & 0,00 \\
\hline Ma/les & $\mathrm{B} / \mathrm{ga}$ & 0,01 & 0,15 & 0,88 & 0,00 & 0,19 & 0,09 & 0,00 & 0,00 & 0,14 & 0,19 & 0,04 & 0,76 & 0,29 & 0,00 \\
\hline Ma/les & Vi/cio & 0,00 & 0,00 & 0,34 & 0,19 & 0,00 & 0,15 & 0,00 & 0,01 & 0,01 & 0,34 & 0,09 & 0,11 & 0,32 & 0,00 \\
\hline Ma/les & Me/lín & 0,13 & 0,95 & 0,29 & 0,01 & 0,67 & 0,14 & 0,02 & 0,00 & 0,00 & 0,00 & 0,03 & 0,81 & 0,00 & 0,00 \\
\hline Ma/les & $\mathrm{Ba} / \mathrm{lla}$ & 0,33 & 0,12 & 0,51 & 0,03 & 0,55 & 0,35 & 0,34 & 0,00 & 0,00 & 0,29 & 0,02 & 0,74 & 0,01 & 0,01 \\
\hline $\mathrm{Ba} / \mathrm{lla}$ & Bo/tá & 0,00 & 0,00 & 0,10 & 0,00 & 0,38 & 0,01 & 0,00 & 0,00 & 0,31 & 0,05 & 0,66 & 0,02 & 0,66 & 0,22 \\
\hline $\mathrm{Ba} / \mathrm{lla}$ & $\mathrm{B} / \mathrm{ga}$ & 0,16 & 0,08 & 0,60 & 0,00 & 0,03 & 0,00 & 0,00 & 0,00 & 0,00 & 0,01 & 0,50 & 0,01 & 0,54 & 0,00 \\
\hline $\mathrm{Ba} / \mathrm{lla}$ & Vi/cio & 0,00 & 0,00 & 0,06 & 0,01 & 0,00 & 0,06 & 0,00 & 0,04 & 0,94 & 0,03 & 0,23 & 0,43 & 0,55 & 0,51 \\
\hline $\mathrm{Ba} / \mathrm{lla}$ & Me/llín & 0,91 & 0,08 & 0,95 & 0,84 & 0,10 & 0,66 & 0,00 & 0,02 & 0,04 & 0,01 & 0,11 & 0,01 & 0,00 & 0,05 \\
\hline $\mathrm{Ba} / \mathrm{lla}$ & Ma/les & 0,00 & 0,00 & 0,61 & 0,04 & 0,03 & 0,24 & 0,00 & 0,54 & 0,36 & 0,84 & 0,17 & 0,02 & 0,00 & 0,92 \\
\hline
\end{tabular}

Ba/lla: Barranquilla, Bo/tá: Bogotá, B/ga: Bucaramanga, Ma/les: Manizales, Me/llín: Medellín, Vi/cio: Villavicencio 
Apuntes CENES Volumen 38, Número 67 enero - junio 2019. Págs. 35-69

\begin{tabular}{|c|c|c|c|c|c|c|c|c|c|c|c|c|c|c|}
\hline Desde & Hacia & P15 & P16 & P17 & P18 & P19 & P20 & P21 & P22 & P23 & P24 & P25 & P26 & P27 \\
\hline Bo/tá & B/ga & 0,26 & 0,21 & 0,02 & 0,40 & 0,15 & & 0,09 & 0,72 & 0,22 & 0,42 & 0,50 & 0,13 & 0,69 \\
\hline Bo/tá & i/cio & 1,00 & 0,11 & 0,43 & 0,12 & 0,86 & 0,96 & 0,08 & 0,80 & 0,96 & 0,63 & 0,00 & 0,46 & 0,06 \\
\hline Bo/tá & Me/lín & 0,00 & 0,02 & 0,64 & 0,89 & 0,10 & 0,00 & 0,61 & 0,02 & 0,47 & 0,28 & 0,08 & 0,07 & 0,68 \\
\hline Bo/tá & Ma/les & 0,00 & 0,79 & 0,27 & 0,77 & 0,74 & 0,53 & 0,07 & 0,48 & 0,69 & 0,30 & 0,32 & 0,44 & 0,26 \\
\hline Bo/tá & $\mathrm{Ba} / \mathrm{lla}$ & 0,01 & 0,48 & 0,00 & 0,18 & 0,01 & 0,48 & 0,90 & 0,61 & 0,81 & 0,45 & 0,45 & 0,38 & 0,01 \\
\hline B/ga & Bo/tá & 0,01 & 0,21 & 0,02 & 0,02 & 0,04 & 0,00 & 0,30 & 0,00 & 0,26 & 0,84 & 0,00 & 0,66 & 0,89 \\
\hline B/ga & Vi/cio & 0,01 & 0,34 & 0,71 & 0,00 & 0,22 & 0,00 & 0,56 & 0,00 & 0,38 & 0,11 & 0,00 & 0,81 & 0,12 \\
\hline B/ga & Me/lín & 0,91 & 0,03 & 0,38 & 0,61 & 0,30 & 0,00 & 0,27 & 0,00 & 0,88 & 0,74 & 0,00 & 0,11 & 0,85 \\
\hline B/ga & Ma/les & 0,86 & 0,16 & 0,62 & 0,10 & 0,67 & 0,01 & 0,00 & 0,01 & 0,18 & 0,51 & 0,10 & 0,59 & 0,40 \\
\hline B/ga & $\mathrm{Ba} / \mathrm{lla}$ & 0,50 & 0,02 & 0,00 & 0,58 & 0,07 & 0,07 & 0,52 & 0,16 & 0,41 & 0,17 & 0,94 & 0,00 & 0,36 \\
\hline Vi/cio & Bo/tá & 0,00 & 0,00 & 0,17 & 0,00 & 0,00 & 0,05 & 0,00 & 0,00 & 0,01 & 0,66 & 0,79 & 0,33 & 0,10 \\
\hline Vi/cio & $\mathrm{B} / \mathrm{ga}$ & 0,07 & 0,04 & 0,15 & 0,03 & 0,82 & 0,01 & 0,01 & 0,00 & 0,01 & 0,54 & 0,00 & 0,17 & 0,71 \\
\hline Vi/cio & Me/lín & 0,02 & 0,01 & 0,19 & 0,21 & 0,65 & 0,00 & 0,07 & 0,00 & 0,50 & 0,90 & 0,33 & 0,17 & 0,99 \\
\hline Vi/cio & Ma/les & 0,04 & 0,42 & 0,88 & 0,52 & 0,76 & 0,02 & 0,97 & 0,00 & 0,36 & 0,96 & 0,45 & 0,19 & 0,25 \\
\hline Vi/cio & $\mathrm{Ba} / \mathrm{lla}$ & 0,01 & 0,70 & 0,00 & 0,82 & 0,04 & 0,28 & 0,37 & 0,02 & 0,43 & 0,00 & 0,83 & 0,02 & 0,04 \\
\hline Me/lín & Bo/tá & 0,10 & 0,06 & 0,02 & 0,01 & 0,98 & 0,04 & 0,00 & 0,00 & 0,52 & 0,96 & 0,20 & 0,80 & 0,05 \\
\hline Me/llín & $\mathrm{B} / \mathrm{ga}$ & 0,09 & 0,11 & 0,02 & 0,01 & 0,91 & 0,31 & 0,00 & 0,63 & 0,75 & 0,81 & 0,01 & 0,62 & 0,46 \\
\hline Me/llín & Vi/cio & 0,72 & 0,01 & 0,41 & 0,01 & 0,13 & 0,56 & 0,17 & 0,41 & 0,84 & 0,05 & 0,53 & 0,58 & 0,03 \\
\hline Me/llín & Ma/les & 0,98 & 0,00 & 0,49 & 0,13 & 0,74 & 0,39 & 0,11 & 0,92 & 0,74 & 0,00 & 0,13 & 0,39 & 0,38 \\
\hline Me/lín & $\mathrm{Ba} / \mathrm{lla}$ & 0,02 & 0,03 & 0,00 & 0,08 & 0,15 & 0,19 & 0,13 & 0,57 & & 0,35 & 0,77 & 0,03 & 0,70 \\
\hline Ma/les & Bo/tá & 0,09 & 0,00 & 0,59 & 0,02 & 0,02 & 0,02 & 0,00 & 0,00 & 0,03 & 0,66 & 0,00 & 0,02 & 0,03 \\
\hline Ma/les & $\mathrm{B} / \mathrm{ga}$ & 0,00 & 0,00 & 0,64 & 0,41 & 0,15 & 0,17 & 0,00 & 0,01 & 0,03 & 0,07 & 0,02 & 0,00 & 0,04 \\
\hline Ma/les & Vi/cio & 0,14 & 0,00 & 0,60 & 0,74 & 0,01 & 0,00 & 0,01 & 0,00 & 0,39 & 0,48 & 0,00 & 0,05 & 0,00 \\
\hline Ma/les & Me/llín & 0,00 & 0,00 & 0,84 & 0,60 & 0,74 & 0,00 & 0,00 & 0,00 & 0,53 & 0,19 & 0,00 & 0,04 & 0,01 \\
\hline Ma/les & $\mathrm{Ba} / \mathrm{lla}$ & 0,03 & 0,00 & 0,26 & 0,48 & 0,24 & 0,88 & 0,70 & 1,00 & 0,49 & 0,02 & 0,44 & 0,32 & 0,16 \\
\hline $\mathrm{Ba} / \mathrm{lla}$ & Bo/tá & 0,70 & 0,03 & 0,01 & 0,00 & 0,00 & 0,15 & 0,05 & 0,36 & 0,08 & 0,23 & 0,02 & 0,00 & 0,00 \\
\hline Ba/lla & $\mathrm{B} / \mathrm{ga}$ & 0,38 & 0,00 & 0,04 & 0,34 & 0,00 & 0,12 & 0,11 & 0,99 & 0,00 & 0,03 & 0,00 & 0,00 & 0,00 \\
\hline Ba/lla & Vi/cio & 0,53 & 0,00 & 0,16 & 0,03 & 0,00 & 0,03 & 0,02 & 0,32 & 0,27 & 0,93 & 0,10 & 0,00 & 0,01 \\
\hline Ba/lla & Me/lín & 0,54 & 0,00 & 0,87 & 0,01 & 0,19 & 0,27 & 0,01 & 0,27 & 0,41 & 0,80 & 0,71 & 0,00 & 0,11 \\
\hline Ba/lla & Ma/les & 0,96 & 0,56 & 0,68 & 0,54 & 0,00 & 0,41 & 0,10 & 0,13 & 0,89 & 0,43 & 0,02 & 0,00 & 0,01 \\
\hline
\end{tabular}

Ba/lla: Barranquilla, Bo/tá: Bogotá, B/ga: Bucaramanga, Ma/les: Manizales, Me/llín: Medellín, Vi/cio: Villavicencio 